\title{
Anthropogenic contaminants of high concern: Existence in water resources and their adverse effects
}

\author{
Itzel Y. López-Pacheco a , Arisbe Silva-Núñez a , Carmen Salinas-Salazar a , Alejandra Arévalo-Gallegos ${ }^{\text {a }}$, \\ Laura A. Lizarazo-Holguin ${ }^{b}$, Damiá Barceló ${ }^{\text {c, d, e }}$, Hafiz M.N. Iqbal ${ }^{\mathrm{a}, ~}$, Roberto Parra-Saldívar ${ }^{\mathrm{a}, ~ *}$ \\ a Tecnologico de Monterrey, School of Engineering and Sciences, Campus Monterrey, Ave. Eugenio Garza Sada 2501, CP 64849 Monterrey, N.L., Mexico \\ ${ }^{\mathrm{b}}$ Universidad de Antioquia, School of Microbiology, Cl. 67 \#53 - 108, Medellín, Antioquia, Colombia \\ c Department of Environmental Chemistry, IDAEA-CSIC, Jordi Girona 18-26, Barcelona 08034, Spain \\ d ICRA, Catalan Institute for Water Research, University of Girona, Emili Grahit 101, Girona 17003, Spain \\ ${ }^{\mathrm{e}}$ Botany and Microbiology Department, College of Science, King Saud University, PO Box 2455, Riyadh 11451, Saudi Arabia
}

\section{A R T I C L E IN F O}

Article history:

Received 22 April 2019

Received in revised form 2 July 2019

Accepted 3 July 2019

Available online xxx

Editor: Patricia Holden

Keywords

Anthropogenic contaminants

Endocrine disruptors

Bioaccumulation

Bio-magnification

Wastewater

Bisphenol A

Toxicity

\begin{abstract}
A B S T R A C T
Existence of anthropogenic contaminants (ACs) in different environmental matrices is a serious and unresolved concern. For instance, ACs from different sectors, such as industrial, agricultural, and pharmaceutical, are found in water bodies with considerable endocrine disruptors potency and can damage the biotic components of the environment. The continuous ACs exposure can cause cellular toxicity, apoptosis, genotoxicity, and alterations in sex ratios in human beings. Whereas, aquatic organisms show bioaccumulation, trophic chains, and biomagnification of ACs through different entry route. These problems have been found in many countries around the globe, making them a worldwide concern. ACs have been found in different environmental matrices, such as water reservoirs for human consumption, wastewater treatment plants (WWTPs), drinking water treatment plants (DWTPs), groundwaters, surface waters, rivers, and seas, which demonstrate their free movement within the environment in an uncontrolled manner. This work provides a detailed overview of ACs occurrence in water bodies along with their toxicological effect on living organisms. The literature data reported between 2017 and 2018 is compiled following inclusion-exclusion criteria, and the obtained information was mapped as per type and source of ACs. The most important ACs are pharmaceuticals (diclofenac, ibuprofen, naproxen, ofloxacin, acetaminophen, progesterone ranitidine, and testosterone), agricultural products or pesticides (atrazine, carbendazim, fipronil), narcotics and illegal drugs (amphetamines, cocaine, and benzoylecgonine), food industry derivatives (bisphenol A, and caffeine), and personal care products (triclosan, and other related surfactants). Considering this threatening issue, robust detection and removal strategies must be considered in the design of WWTPs and DWTPs.
\end{abstract}

\section{Introduction}

Anthropogenic contaminants (ACs) are substances found in the environment due to human activities (Rhind, 2009), and can effects the living organisms, directly or indirectly. Many of these contaminants have now been recognized as endocrine disruptors and can pose human-health related risks, such as hormonal imbalance, metabolism disorders, neurological disorders, immunological disorders, male/ female reproductive system imbalance (Garcia-Morales et al., 2015; Barrios-Estrada et al., 2018a; Bilal et al., 2019a). Therefore, ACs containing wastewater treatment methods are of great importance due to the concentration and variety of emerging pollutants derived from anthropogenic processes (Rodríguez-Delgado et al., 2016; Bilal et al., 2017; Barrios-Estrada et al., 2018b; Bilal and Iqbal, 2019; Bilal et al.,

\footnotetext{
* Corresponding authors.

Email addresses: hafiz.iqbal@tec.mx (H.M.N. Iqbal); r.parra@tec.mx (R.
} Parra-Saldívar) 2019b; Bilal et al., 2019c; López-Pacheco et al., 2019). The maximum concentrations of ACs detected in water bodies are shown in Fig. 1. Based on a data reported in Scopus from 2017 to 2018, ACs of high concern from different countries, types, and sources are summarized in Table 1. Some of these contaminants cannot be metabolized easily and thus accumulate in living organisms. From a broader perspective, such accumulation of concerning agents in living species is known as bioaccumulation. Whereas, the passage of the contaminant through various levels of a trophic chain is known as biomagnification (Blowes et al., 2003). The controlled or uncontrolled bioaccumulation and biomagnification of ACs can cause several adverse effects on living beings (Fig. 2).

There are many reports that confirm the persistence/existence of $\mathrm{ACs}$ in water bodies of several countries around the world. For instance, the sampling over two periods, i.e., (1) February 2014 and (2) October 2014, was performed in the Guarapiranga reserve in Brazil. In the first period, $31 \mathrm{ACs}$ were detected, while in the second, around 27 ACs were recorded in the Guarapiranga reserve (López-Doval et 


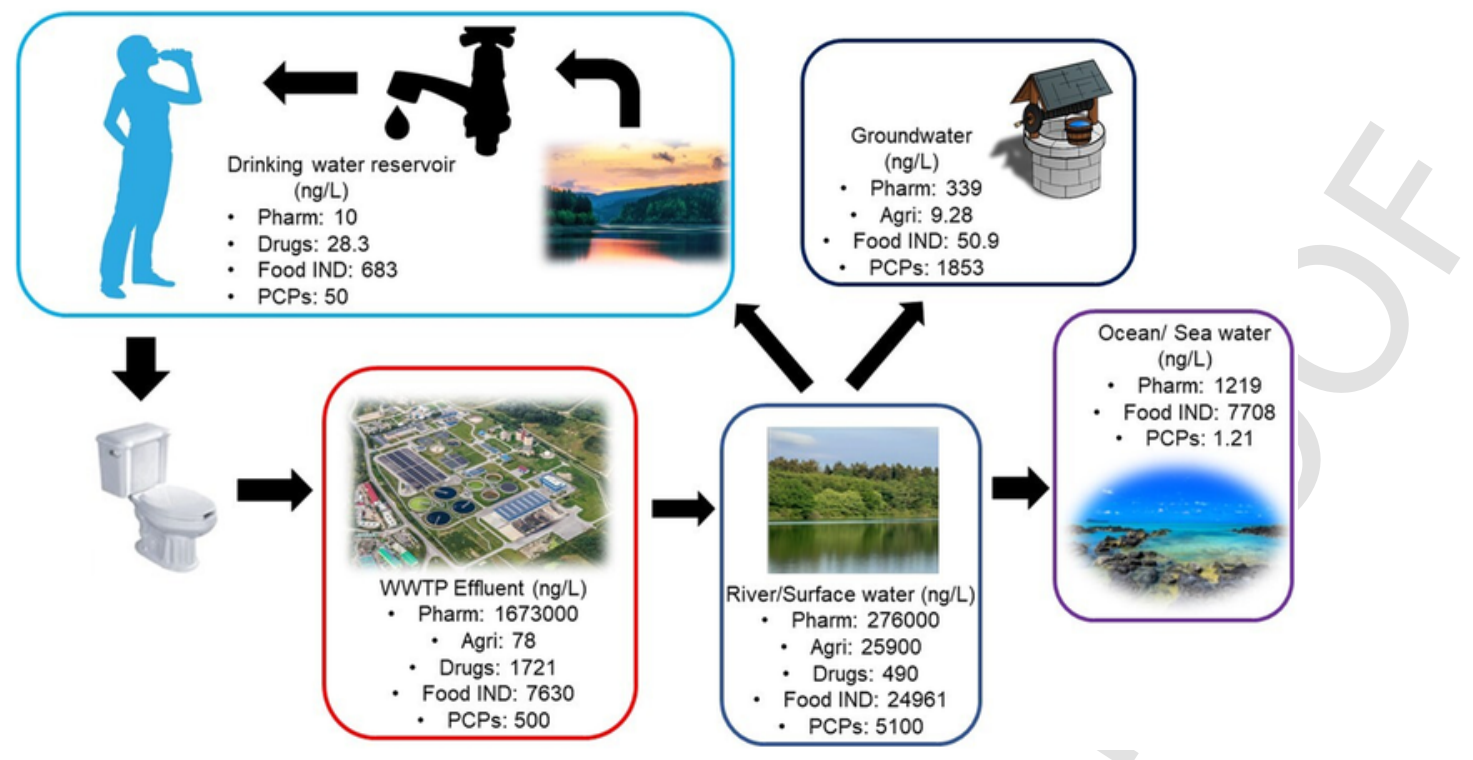

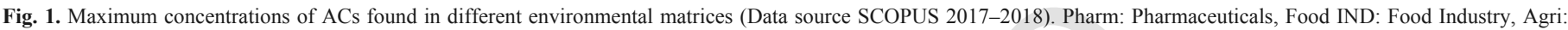
Agricultural. PCPs: Personal care products.

Table 1

Range (ng/L) of ACs reported in 2017 and 2018 in Scopus. The obtained data is summarized as per source and type of ACs.

\begin{tabular}{|c|c|c|c|}
\hline Source & Type & Range (ng/L) & $\begin{array}{l}\text { \# of reported } \\
\text { countries }\end{array}$ \\
\hline \multirow[t]{5}{*}{ Drinking water } & Pharmaceuticals & 10.3 & 1 \\
\hline & Drugs & $0.61-28.3$ & 1 \\
\hline & Food industry & $2.4-683$ & 1 \\
\hline & Personal Care & $1.1-50$ & 1 \\
\hline & Products & & \\
\hline \multirow[t]{6}{*}{ WTTP effluent } & Pharmaceuticals & $0.103-1,673,000$ & 9 \\
\hline & Agricultural & 78 & 1 \\
\hline & Drugs & $50-1721$ & 3 \\
\hline & Food industry & $2.4-7630$ & 4 \\
\hline & Personal Care & $<0.6-500$ & 4 \\
\hline & Products & & \\
\hline \multirow{6}{*}{$\begin{array}{l}\text { River/surface } \\
\text { water }\end{array}$} & Pharmaceuticals & $0.11-276,000$ & 15 \\
\hline & Agricultural & $1-25,900$ & 5 \\
\hline & Drugs & $4-490$ & 2 \\
\hline & Food industry & $1.7-24,961$ & 7 \\
\hline & Personal Care & $0.4-5100$ & 8 \\
\hline & Products & & \\
\hline \multirow[t]{4}{*}{ Ocean/sea water } & Pharmaceuticals & $0.0038-1219$ & 5 \\
\hline & Food industry & $0.03-7708$ & 3 \\
\hline & Personal Care & $0.0036-1.21$ & 1 \\
\hline & Products & & \\
\hline \multirow[t]{5}{*}{ Groundwater } & Pharmaceuticals & $0.33-339$ & 2 \\
\hline & Agricultural & 9.28 & 1 \\
\hline & Food industry & 50.9 & 1 \\
\hline & Personal Care & $3.7-1853$ & 2 \\
\hline & Products & & \\
\hline
\end{tabular}

al., 2017). From the Holtemme river in Germany, a study showed the presence of 86 micropollutants. Out of the total 86 , around 50 ACs were detected in the water sample, around 47 in the sediment and 17 in the specimens of Gammarus pulex (Inostroza et al., 2017). Other studies also report the presence of ACs in the water reservoir, WWTPs, and vegetated draining ditch in Brazil and Mexico (Estrada-Arriaga et al., 2016; López-Doval et al., 2017; Moeder et al., 2017). The main ACs reported in different studies are pharmaceuticals (diclofenac, ibuprofen, naproxen, ofloxacin, acetaminophen, progesterone ranitidine and testosterone), agricultural products or pesticides (atrazine, carbendazim, and fipronil), narcotics and illegal drugs (amphetamines, cocaine, and benzoylecgonine), food industry derivatives (bisphenol A, caffeine), and personal care products (triclosan, and other related surfactants). However, regardless of their concerning risk, there is no single report available in the literature that discusses all of them at one place with suitable examples. Thus, herein, an effort has been made to fill this literature gap. In addition, various environmentally related matrices, such as water reservoirs, wastewater treatment plants (WWTPs), drinking water treatment plants (DWTPs), groundwaters, surface waters, rivers, and seas, in which ACs were found, are discussed with suitable examples. Following a detailed inclusion-exclusion criterion, the Scopus dataset from the year 2017-2018 was scrutinized and comprehensively summarized in Table 2. Moreover, the obtained information was also mapped using the software ArcGIS 9.3.1 (Esri, USA), as per type and source of ACs (Fig. 3).

\section{Contaminants from pharmaceutical products}

\subsection{Diclofenac}

Diclofenac is a non-steroidal anti-inflammatory drug (NSAID) (Wang et al., 2010). In Europe, diclofenac has been found and reported in many water bodies. In Portugal, concentrations of $972 \mathrm{ng} / \mathrm{L}$ were found in WWTPs and rivers (Paíga et al., 2016). In Spain, diclofenac concentration ranged from 1 to $54 \mathrm{ng} / \mathrm{L}$ (Silva et al., 2011). More specifically, in the Turia River Basin (Valencia, Spain), diclofenac was found from 6.72 to $940 \mathrm{ng} / \mathrm{L}$ (Carmona et al., 2014). In Latin America (Cuernavaca, Mexico), concentrations between 258 and 1398, ng/L were detected (Rivera-Jaimes et al., 2018). In Chinese rivers, a maximum of $717 \mathrm{ng} / \mathrm{L}$ diclofenac was found (Wang et al., 2010). In Pakistan, the concentrations were found in the range of 10 to $1800 \mathrm{ng} / \mathrm{L}$ (Scheurell et al., 2009). In a WWTP in Turkey, the influent had a diclofenac concentration of $295-1376 \mathrm{ng} / \mathrm{L}$, and the effluent had 119-1012 ng/L, resulting in removal efficiencies from 26 to $60 \%$ (Sari et al., 2014). In Malaysia, diclofenac in living organisms, from a river Estuary, was detected in fishes and mollusks samples $(1.42 \mathrm{ng} / \mathrm{g}$ to $10.76 \mathrm{ng} / \mathrm{g}$ of diclofenac) (Omar et al., 2019). Regarding toxicological effects in fauna, it has been found that exposure 


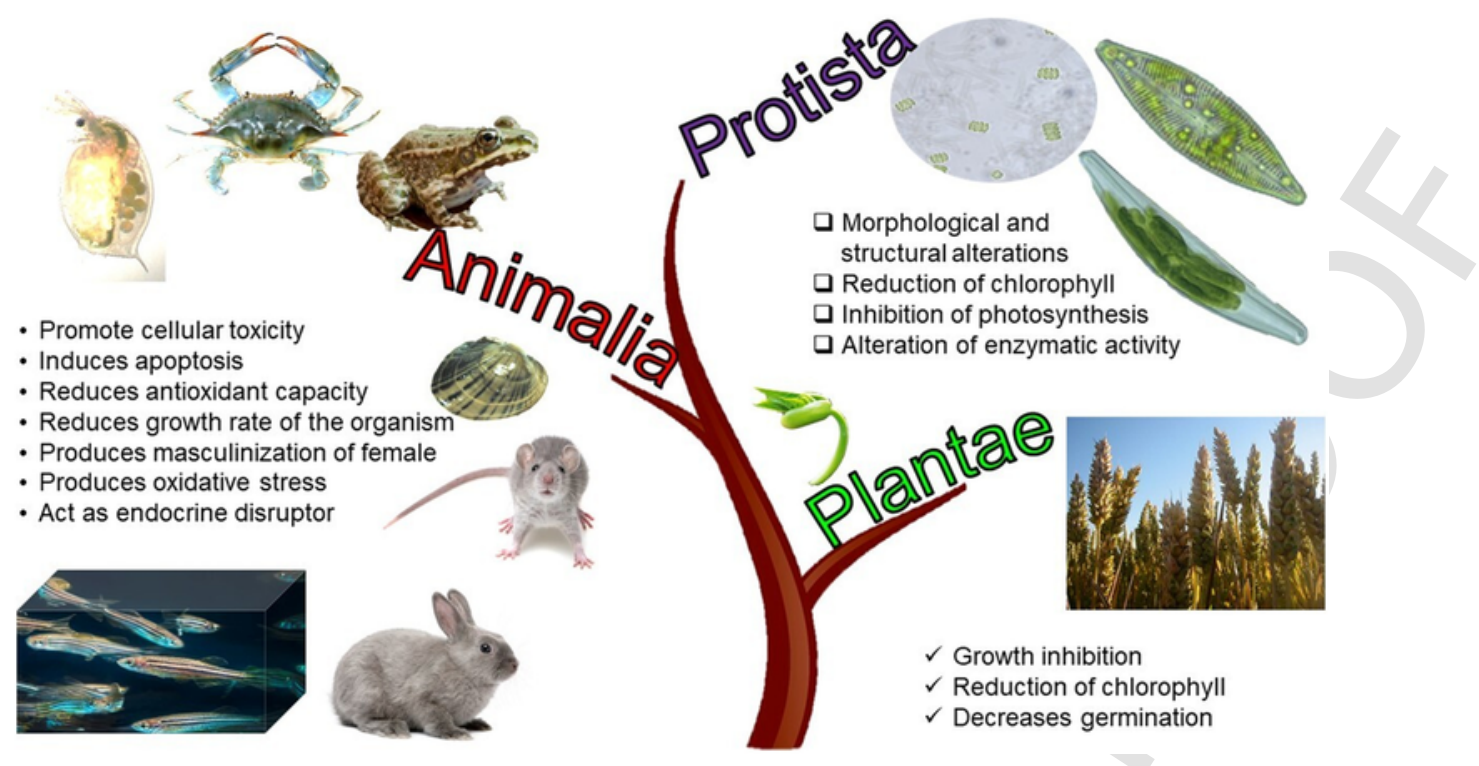

Fig. 2. Adverse effects of ACs on living organisms in a biological kingdom.

to diclofenac (200, 2000 and 20,000 ng/L) affects liver activity, decreases lipid peroxidation and reduces the amount of secreted dopamine in the fish Rhamdia quelen (Guiloski et al., 2017b). In the freshwater crustaceans, Daphnia magna, and Moina macrocopa, a reduction in the reproductive rate was observed at a concentration higher than $25 \mathrm{mg} / \mathrm{L}$ (Lee et al., 2011). Diclofenac affects the cellular level in the fish Oryzias latipes and causes cellular toxicity, apoptosis, genotoxicity and estrogenic effects at the concentrations of $8 \mathrm{ng} /$ $\mathrm{L}$ and $1000 \mathrm{ng} / \mathrm{L}$ (Hong et al., 2007). Diclofenac can be bio-accumulated and metabolized by animals. For instance, the mussels Mytilus trossulus can metabolize diclofenac into its hydroxy-derivative compounds (4-OH and 5-OH diclofenac) (Świacka et al., 2019). Furthermore, diclofenac affects amphibians, by producing morphological abnormalities, and alterations in the cardiac function and swimming performance (Peltzer et al., 2019).

\subsection{Ibuprofen}

Ibuprofen is an NSAID and an analgesic (Moro et al., 2014). It can be detected as a whole or in parts as metabolites, such as hydroxyibuprofen and carboxyibuprofen, in water bodies (Dvořáková Březinova et al., 2018). Ibuprofen has been reported at the concentration of $13.74 \mu \mathrm{g} / \mathrm{L}$ and its metabolites at the concentration of $130 \mu \mathrm{g} / \mathrm{L}$, in WWTPs in Spain (Ferrando-Climent et al., 2012). In South Africa, ibuprofen was found at the concentrations of 278, 261, and $170 \mathrm{ng} /$ $\mathrm{L}$, in the water samples from Estuary and seawater (Primrose et al., 2019). In Cameroon, the samples from surface water and groundwater contained ibuprofen at $516 \mathrm{ng} / \mathrm{L}$ and $276 \mathrm{ng} / \mathrm{L}$, respectively (Branchet et al., 2019). In the UK, ibuprofen was found in surface water at $6297 \mathrm{ng} / \mathrm{L}$ (Letsinger et al., 2019). Exposure to ibuprofen $(1 \mathrm{mg} / \mathrm{L})$ can cause a reduction in the growth rate of microorganisms. It can also induce morphological and structural alterations, including a reduction in chlorophyll production and an increase in the production of carotenoids (Moro et al., 2014). In Navicula sp. extended time exposure (10 days) to ibuprofen at the concentrations from 10 to $100 \mathrm{mg} / \mathrm{L}$ inhibits the photosynthesis rate of the diatom (T. Ding et al., 2017b). In the frog Pelophylax ridibundus, ibuprofen $(250 \mathrm{ng} / \mathrm{L})$ elevates oxyradicals and produces instability of the lysosomal membrane (Falfushynska et al., 2017). In zebrafish (Danio re- rio), exposure to ibuprofen ( 5 to $500 \mu \mathrm{g} / \mathrm{L}$ ) reduces the growth rate, reduces the ability to respond to external stimuli and movement, and neurotoxic to the embryos (Xia et al., 2017). In zebra mussels, Dreissena polymorpha, ibuprofen $(100 \mu \mathrm{g} / \mathrm{L})$ increases the oxidation of lipids, decreases the amount of triglycerides, and antioxidant capacity (André and Gagné, 2017).

\subsection{Naproxen}

Naproxen is an analgesic and extensively used to treat moderate pain, fever, headache, and inflammation (Neal and Moore, 2017). While, it is considered as a toxic compound for some species, such as Pseudokirchneriella subcapitata, Brachionus calyciflorus and Ceriodaphnia dubia, subject to chronic exposure. The concentrations up to $31.81 \mathrm{mg} / \mathrm{L}, 0.56 \mathrm{mg} / \mathrm{L}$, and $0.33 \mathrm{mg} / \mathrm{L}$, and its photo-derivatives are considered even toxic than the original molecule in the above-mentioned species (Isidori et al., 2005). In Algiers, on the west side of Mediterranean Sea bay, naproxen has been found at the concentrations between 1220 and $9585 \mathrm{ng} / \mathrm{L}$ in wastewater, and $228.3 \mathrm{ng} / \mathrm{L}$ in the surface water (Kermia et al., 2016). In Kinmen (Taiwan), naproxen was present at the concentration of $0.3 \mathrm{ng} / \mathrm{L}$ in Taihu Lake and 104.3 ng/L in WWTP (Wei-po Lai et al., 2016). Also, it was found from 52.4 to $124.2 \mathrm{ng} / \mathrm{L}$ in surface water in Italy (Riva et al., 2019). In Pakistan, 215 to $464 \mu \mathrm{g} / \mathrm{L}$ of this pollutant had been found in pharmaceutical industry wastewater effluents (Ashfaq et al., 2017). The crayfish Orconectes virilis has been used to assess the effects of naproxen on marine species. The concentrations of $0.027 \mu \mathrm{g} / \mathrm{L}, 2.30 \mu \mathrm{g} / \mathrm{L}$, and $14.0 \mu \mathrm{g} / \mathrm{L}$ showed a negative effect on the behavior and motility of marine species (Neal and Moore, 2017). Carps exposed at different concentrations of naproxen $(10,50,100$ and $200 \mu \mathrm{g} / \mathrm{L})$ in their early stages of development, showed alterations in the rate of development, morphology, histopathology, and in some cases, increase in the mortality of organisms (Sehonova et al., 2017). In some microorganisms, such as microalgae Cymbella sp. and Scenedesmus quadricauda, it has been proven that this compound at 50 and $100 \mu \mathrm{g} / \mathrm{L}$ causes alterations to the amount of chlorophyll, carotenoids, and enzymatic activity (T. Ding et al., 2017a). In adult zebrafishes (Danio rerio), naproxen ( 1 and $100 \mathrm{mg} / \mathrm{L})$ causes an alter- 
Table 2

Anthropogenic contaminants (ACs) reported in wastewater treatment plants effluent and water resources in 2017 and 2018 (SCOPUS database).

\begin{tabular}{|c|c|c|c|c|}
\hline Contaminant & Location & Source & Concentration (ng/L) & Reference \\
\hline \multicolumn{5}{|l|}{ Pharmaceuticals } \\
\hline \multirow[t]{14}{*}{ Acetaminophen } & Antarctic Peninsula & Stream & 38 & (González-Alonso et al., 2017) \\
\hline & Canada & WWTP & $150-570$ & (Brown and Wong, 2018) \\
\hline & \multirow[t]{5}{*}{ China } & WWTP & $2.9-58.4$ & (Zhang et al., 2018) \\
\hline & & River & $3.1-13.7$ & (He et al., 2018) \\
\hline & & WWTP & 39.8 & (Wang et al., 2018) \\
\hline & & Surface water & 75 & (Yao et al., 2018) \\
\hline & & River & 1490 & (Zha et al., 2017) \\
\hline & Colombia & WWTP & $25-35,100$ & (Botero-coy et al., 2018) \\
\hline & Iran & WWTP & $17-441$ & (Biel-maeso et al., 2018b) \\
\hline & Italy & River & 226 & (Mandaric et al., 2017) \\
\hline & Korea & Coastal area & 48 & (Kim et al., 2017) \\
\hline & Saudi Arabia & Sea water & 2363 & (Ali et al., 2017) \\
\hline & Spain & Sea water & 41.5 & (Biel-maeso et al., 2018a) \\
\hline & Taiwan & Aquaculture ponds & 91 & (Lai et al., 2018) \\
\hline Acyclovir & Great Lakes & Lake & 1100 & (Elliott et al., 2018) \\
\hline Amantadine & China & Surface water & $108.5-1785$ & (Peng et al., 2018) \\
\hline Amitriptyline & China & River & 4.8 & (Wu et al., 2017) \\
\hline Ampicillin & Vietnam & River & $40-164$ & (Thai et al., 2018) \\
\hline Aspirin & India & River & 1340 & (Mutiyar et al., 2018) \\
\hline \multirow[t]{9}{*}{ Atenolol } & Brazil & Surface water & $12.6-665$ & (Ribeiro de Sousa et al., 2018) \\
\hline & France & Treatment wetlands & 1260 & (Nuel et al., 2018) \\
\hline & Iran & WWTP & $134-2110$ & (Biel-maeso et al., 2018b) \\
\hline & Italy & River & 18.1 & (Mandaric et al., 2017) \\
\hline & Korea & Coastal area & 85.7 & (Kim et al., 2017) \\
\hline & \multirow[t]{2}{*}{ Spain } & Sea water & 138.9 & (Biel-maeso et al., 2018a) \\
\hline & & WWTP & 211 & (Afonso-Olivares et al., 2017) \\
\hline & United Kingdom & River & $10.1-100$ & (Burns et al., 2018) \\
\hline & USA & Surface water & 1700 & (Elliott et al., 2018) \\
\hline Atorvastatin & Italy & River & 21.7 & (Mandaric et al., 2017) \\
\hline \multirow[t]{6}{*}{ Azithromycin } & China & WWTP & $88-680$ & (Lin et al., 2018b) \\
\hline & Colombia & WWTP & $3020-4120$ & (Botero-coy et al., 2018) \\
\hline & Portugal & River & $32.12-35.66$ & (Pereira et al., 2017) \\
\hline & Spain & Sea water & 17.8 & (Biel-maeso et al., 2018a) \\
\hline & & Groundwater & $4.86-13.01$ & (Boy-roura et al., 2018) \\
\hline & Vietnam & River & $19-2270$ & (Thai et al., 2018) \\
\hline \multirow[t]{4}{*}{ Bezafibrate } & China & Groundwater & 0.33 & (L. Ma et al., 2018) \\
\hline & & Landfill leachates & 660 & (Sui et al., 2017) \\
\hline & Italy & River & 10.32 & (Mandaric et al., 2017) \\
\hline & Spain & WWTP & 260 & (Afonso-Olivares et al., 2017) \\
\hline Bromazepam & China & Surface water & $2.58-3.72$ & (Xiang et al., 2018) \\
\hline \multirow[t]{28}{*}{ Carbamazepine } & Bangladesh & River & 8.8 & (Hossain et al., 2018) \\
\hline & Brazil & Surface water & $12.6-659$ & (Ribeiro de Sousa et al., 2018) \\
\hline & China & WWTP & $43.4-672.5$ & (Zhang et al., 2018) \\
\hline & & WWTP & $0.268-57.8$ & (Wang et al., 2018) \\
\hline & & Groundwater & $0.42-1.21$ & (L. Ma et al., 2018) \\
\hline & & Surface water & 69 & (Yao et al., 2018) \\
\hline & & Surface water & $12.28-83.8$ & (Xiang et al., 2018) \\
\hline & & Groundwater & 18.1 & (Yang et al., 2018) \\
\hline & & Surface water & 9.78 & (Yang et al., 2018) \\
\hline & & Landfill leachates & $2120-6270$ & (Sui et al., 2017) \\
\hline & & River & 13.9 & (Zha et al., 2017) \\
\hline & & Surface water & $1.38-145$ & (Peng et al., 2018) \\
\hline & France & Seawater & 1.01 & (Poi et al., 2018) \\
\hline & & Treatment wetlands & 448 & (Nuel et al., 2018) \\
\hline & Great Lakes & Lake & 330 & (Elliott et al., 2018) \\
\hline & Hungary & Surface water & $60-276,000$ & (Bókony et al., 2018) \\
\hline & India & River & 1346 & (Mutiyar et al., 2018) \\
\hline & Iran & WWTP & $21-657$ & (Biel-maeso et al., 2018b) \\
\hline & Italy & Drinking water & 10.3 & (Riva et al., 2018) \\
\hline & & River & 137 & (Mandaric et al., 2017) \\
\hline & Korea & WWTP & $1035-11,478$ & (Ibe et al., 2018) \\
\hline & & River & $14-2900$ & (Ibe et al., 2018) \\
\hline & & Coastal area & $4.58-38.6$ & (Kim et al., 2017) \\
\hline & Saudi Arabia & Sea water & 110 & (Ali et al., 2017) \\
\hline & Spain & Sea water & 31.1 & (Biel-maeso et al., 2018a) \\
\hline & & WWTP & 1290 & (Afonso-Olivares et al., 2017) \\
\hline & United Kingdom & River & $8.7-195$ & (Burns et al., 2018) \\
\hline & USA & Surface water & 330 & (Elliott et al., 2018) \\
\hline
\end{tabular}


Table 2 (Continued)

\begin{tabular}{|c|c|c|c|c|}
\hline Contaminant & Location & Source & Concentration (ng/L) & Reference \\
\hline Cefalexin & Italy & River & 17.1 & (Mandaric et al., 2017) \\
\hline Cefotaxim & Vietnam & River & $10-145$ & (Thai et al., 2018) \\
\hline Cefuroxim & Vietnam & River & $195-7860$ & (Thai et al., 2018) \\
\hline Celestolide & Italy & River & 74.3 & (Mandaric et al., 2017) \\
\hline Cimetidine & United Kingdom & River & 44 & (Burns et al., 2018) \\
\hline \multirow[t]{8}{*}{ Ciprofloxacin } & China & WWTP & $2.54-26.2$ & (Wang et al., 2018) \\
\hline & Colombia & WWTP & $446-1070$ & (Botero-coy et al., 2018) \\
\hline & Iran & WWTP & $48-1450$ & (Biel-maeso et al., 2018b) \\
\hline & Korea & Coastal area & 1.25 & (Kim et al., 2017) \\
\hline & Spain & Sea water & 211 & (Biel-maeso et al., 2018a) \\
\hline & & WWTP & 89 & (Afonso-Olivares et al., 2017) \\
\hline & & Groundwater & $30.04-298.29$ & (Boy-roura et al., 2018) \\
\hline & Vietnam & River & $75-40,900$ & (Thai et al., 2018) \\
\hline \multirow[t]{4}{*}{ Citalopram } & China & River & 5.1 & (Wu et al., 2017) \\
\hline & Italy & & 93 & (Mandaric et al., 2017) \\
\hline & Portugal & & $20.70-52.97$ & (Pereira et al., 2017) \\
\hline & United Kingdom & & 71.4 & (Burns et al., 2018) \\
\hline Clarithromycin & Antarctic Peninsula & Glacier drain & 20 & (González-Alonso et al., 2017) \\
\hline \multirow[t]{6}{*}{ Clarithromycin } & China & WWTP & $1.2-342$ & (Zhang et al., 2018) \\
\hline & & & $87-160$ & (Lin et al., 2018b) \\
\hline & Iran & & 7640 & (Biel-maeso et al., 2018b) \\
\hline & Italy & River & 159 & (Mandaric et al., 2017) \\
\hline & Portugal & & $24.8-39.1$ & (Pereira et al., 2017) \\
\hline & Vietnam & & $10-55,097$ & (Thai et al., 2018) \\
\hline \multirow[t]{2}{*}{ Climbazole } & China & Groundwater & 67.7 & (Yang et al., 2018) \\
\hline & & Surface water & 276 & (Yang et al., 2018) \\
\hline Clofibric acid & China & Groundwater & 0.85 & (L. Ma et al., 2018) \\
\hline Clomipramine & China & River & 3.2 & (Wu et al., 2017) \\
\hline \multirow{2}{*}{ Clotrimazole } & China & Surface water & 13.6 & (Yang et al., 2018) \\
\hline & & Groundwater & 3.23 & (Yang et al., 2018) \\
\hline \multirow[t]{3}{*}{ Codeine } & India & River & 262 & (Mutiyar et al., 2018) \\
\hline & Italy & & 40.04 & (Mandaric et al., 2017) \\
\hline & United Kingdom & & $8.0-101$ & (Burns et al., 2018) \\
\hline Coprostanol & Vietnam & River & 57,800 & (Chau et al., 2018) \\
\hline Cyclophosphamid & China & WWTP & $0.103-3.20$ & (Wang et al., 2018) \\
\hline Danofloxacin & Spain & Groundwater & $26.39-67.78$ & (Boy-roura et al., 2018) \\
\hline \multirow[t]{2}{*}{ Desvenlafaxine } & Great Lakes & Lake & 1200 & (Elliott et al., 2018) \\
\hline & United Kingdom & River & $4.6-268$ & (Burns et al., 2018) \\
\hline \multirow[t]{3}{*}{ Diazepam } & China & Surface water & $15.26-79.33$ & (Xiang et al., 2018) \\
\hline & & & $2.5-104$ & (Peng et al., 2018) \\
\hline & India & River & 305 & (Mutiyar et al., 2018) \\
\hline \multirow[t]{25}{*}{ Diclofenac } & Antarctic Peninsula & Stream & 7761 & (González-Alonso et al., 2017) \\
\hline & & Glacier drain & 77 & \\
\hline & Brazil & Surface water & $4.8-364$ & (Ribeiro de Sousa et al., 2018) \\
\hline & China & River & 32 & (Lin et al., 2018a) \\
\hline & & WWTP & $7.9-237.7$ & (Zhang et al., 2018) \\
\hline & & River & 20.2 & (He et al., 2018) \\
\hline & & Groundwater & $0.84-1.87$ & (L. Ma et al., 2018) \\
\hline & & Surface water & 180 & (Yao et al., 2018) \\
\hline & & Groundwater & 6.03 & (Yang et al., 2018) \\
\hline & & Surface water & 45.3 & (Yang et al., 2018) \\
\hline & & WWTP & $13-59$ & (Lin et al., 2018b) \\
\hline & & Landfill leachates & $4810-19,300$ & (Sui et al., 2017) \\
\hline & & River & 374 & (Zha et al., 2017) \\
\hline & Czech Republic & Surface water & 1070 & (Marsik et al., 2017) \\
\hline & France & Treatment Wetlands & 7377 & (Nuel et al., 2018) \\
\hline & Germany & Surface water & $1.2-486.5$ & (Fisch et al., 2017) \\
\hline & Iran & WWTP & $38-1020$ & (Biel-maeso et al., 2018b) \\
\hline & Italy & River & 675 & (Mandaric et al., 2017) \\
\hline & Mediterranean Sea & Sea water & 0.02 & (Brumovský et al., 2017) \\
\hline & Pakistan & WWTP & 836,000 & (Ashfaq et al., 2017) \\
\hline & Portugal & River & $25.13-51.24$ & (Pereira et al., 2017) \\
\hline & Saudi Arabia & Sea water & 14,020 & (Ali et al., 2017) \\
\hline & Slovenia & WWTP & $1.24-25.3$ & (Cesen et al., 2018) \\
\hline & & River & $1.81-158$ & (Cesen et al., 2018) \\
\hline & Spain & Sea water & 31.9 & (Biel-maeso et al., 2018a) \\
\hline Diltiazem & Italy & River & 10.5 & (Mandaric et al., 2017) \\
\hline Dimetridazole & China & Surface water & 110 & (Yao et al., 2018) \\
\hline Doxycycline & China & River & 32.9 & (He et al., 2018) \\
\hline
\end{tabular}


Table 2 (Continued)

\begin{tabular}{|c|c|c|c|c|}
\hline Contaminant & Location & Source & Concentration (ng/L) & Reference \\
\hline Enrofloxacin & China & River & 2.9 & (He et al., 2018) \\
\hline \multirow[t]{11}{*}{ Erythromycin } & China & WWTP & $2.4-271.3$ & (Zhang et al., 2018) \\
\hline & & Surface water & 9.2 & (Yao et al., 2018) \\
\hline & & Groundwater & 57.6 & (Yang et al., 2018) \\
\hline & & WWTP & $25-99$ & (Lin et al., 2018b) \\
\hline & Iran & WWTP & $18-359$ & (Biel-maeso et al., 2018b) \\
\hline & Italy & River & 91.9 & (Mandaric et al., 2017) \\
\hline & Korea & Coastal area & 0.196 & (Kim et al., 2017) \\
\hline & Portugal & River & $32.89-38.8$ & (Pereira et al., 2017) \\
\hline & Spain & Sea water & 2.3 & (Biel-maeso et al., 2018a) \\
\hline & China & Surface water & 425 & (Yang et al., 2018) \\
\hline & Taiwan & Aquaculture ponds & $5.5-57.4$ & (Lai et al., 2018) \\
\hline Estazolam & China & Surface water & $0.53-1.06$ & (Xiang et al., 2018) \\
\hline Fenbendazole & Korea & Coastal area & $0.487-9.69$ & (Kim et al., 2017) \\
\hline \multirow[t]{2}{*}{ Fenoprofen } & France & Treatment Wetlands & 2481 & (Nuel et al., 2018) \\
\hline & Spain & Sea water & 7.5 & (Biel-maeso et al., 2018a) \\
\hline Fexofenadine & USA & Surface water & 3600 & (Elliott et al., 2018) \\
\hline \multirow[t]{2}{*}{ Fluconazole } & China & Groundwater & 75.6 & (Yang et al., 2018) \\
\hline & & Surface water & 121 & (Yang et al., 2018) \\
\hline Flumequine & Taiwan & Aquaculture ponds & $1.8-331$ & (Lai et al., 2018) \\
\hline \multirow[t]{3}{*}{ Fluoxetine } & China & WWTP & $2.36-24.8$ & (Wang et al., 2018) \\
\hline & & River & $2.3-42.9$ & (Wu et al., 2017) \\
\hline & Iran & WWTP & $4-1570$ & (Biel-maeso et al., 2018b) \\
\hline \multirow[t]{2}{*}{ Furosemide } & Iran & WWTP & $161-1990$ & (Biel-maeso et al., 2018b) \\
\hline & Italy & River & 359 & (Mandaric et al., 2017) \\
\hline \multirow[t]{2}{*}{ Gabapentin } & United Kingdom & River & $17.4-1445$ & (Burns et al., 2018) \\
\hline & Vietnam & WWTP & $690-1700$ & (Nguyen et al., 2018) \\
\hline \multirow[t]{3}{*}{ Gemfibrozil } & Iran & WWTP & $518-3720$ & (Biel-maeso et al., 2018b) \\
\hline & Italy & River & 19.1 & (Mandaric et al., 2017) \\
\hline & China & Landfill leachates & $2010-4480$ & (Sui et al., 2017) \\
\hline Hydrochlorothiazide & Antarctic Peninsula & Glacier drain & 19 & (González-Alonso et al., 2017) \\
\hline \multirow[t]{2}{*}{ Hydrochlorothiazide } & Iran & WWTP & $280-4430$ & (Biel-maeso et al., 2018b) \\
\hline & Italy & River & 189.5 & (Mandaric et al., 2017) \\
\hline \multirow[t]{22}{*}{ Ibuprofen } & Antarctic Peninsula & Stream & 974 & (González-Alonso et al., 2017) \\
\hline & Brazil & Surface water & $6.75-373$ & (Ribeiro de Sousa et al., 2018) \\
\hline & China & River & $2.4-320$ & (Lin et al., 2018a) \\
\hline & & River & 14.3 & (He et al., 2018) \\
\hline & & WWTP & $26.4-294$ & (Wang et al., 2018) \\
\hline & & Surface water & 590 & (Yao et al., 2018) \\
\hline & & WWTP & $52-100$ & (Lin et al., 2018b) \\
\hline & & River & 203 & (Zha et al., 2017) \\
\hline & Czech Republic & Surface water & 3210 & (Marsik et al., 2017) \\
\hline & France & Treatment Wetlands & 3129 & (Nuel et al., 2018) \\
\hline & India & River & 2302 & (Mutiyar et al., 2018) \\
\hline & Iran & WWTP & $95-751$ & (Biel-maeso et al., 2018b) \\
\hline & Italy & River & 116 & (Mandaric et al., 2017) \\
\hline & Mediterranean Sea & Sea water & $0.063-1.08$ & (Brumovský et al., 2017) \\
\hline & Pakistan & WWTP & $1,673,000$ & (Ashfaq et al., 2017) \\
\hline & Saudi Arabia & Sea water & 509 & (Ali et al., 2017) \\
\hline & Spain $>$ & Sea water & 1219 & (Biel-maeso et al., 2018a) \\
\hline & & WWTP & 21,700 & (Afonso-Olivares et al., 2017) \\
\hline & China & Groundwater & 48.7 & (Yang et al., 2018) \\
\hline & & Surface water & 292 & (Yang et al., 2018) \\
\hline & Slovenia & WWTP & $1.82-35.9$ & (Cesen et al., 2018) \\
\hline & & River & $1.44-46.1$ & (Cesen et al., 2018) \\
\hline \multirow[t]{3}{*}{ Indomethacin } & Czech Republic & Surface water & 69.29 & (Marsik et al., 2017) \\
\hline & Italy & River & 28.5 & (Mandaric et al., 2017) \\
\hline & Antarctic Peninsula & Glacier drain & 56 & (González-Alonso et al., 2017) \\
\hline Irbesartan & Italy & River & 149 & (Mandaric et al., 2017) \\
\hline \multirow[t]{10}{*}{ Ketoprofen } & Brazil & River & 620 & (Honjo et al., 2017) \\
\hline & China & WWTP & $19.7-844$ & (Wang et al., 2018) \\
\hline & Czech Republic & Surface water & 930 & (Marsik et al., 2017) \\
\hline & France & Seawater & 1.56 & (Poi et al., 2018) \\
\hline & & Treatment wetlands & 319 & (Nuel et al., 2018) \\
\hline & Iran & WWTP & $210-5480$ & (Biel-maeso et al., 2018b) \\
\hline & Italy & River & 193 & (Mandaric et al., 2017) \\
\hline & Mediterranean Sea & Sea water & 0.179 & (Brumovský et al., 2017) \\
\hline & Spain & WWTP & 1170 & (Afonso-Olivares et al., 2017) \\
\hline & Taiwan & Aquaculture ponds & $8.3-24.7$ & (Lai et al., 2018) \\
\hline
\end{tabular}


Table 2 (Continued)

\begin{tabular}{|c|c|c|c|c|}
\hline Contaminant & Location & Source & Concentration (ng/L) & Reference \\
\hline Levamisole & Italy & River & 9.44 & (Mandaric et al., 2017) \\
\hline \multirow[t]{4}{*}{ Lidocaine } & Great Lakes & Lake & 2100 & (Elliott et al., 2018) \\
\hline & USA & Surface water & $0.6-431$ & (Radley et al., 2017) \\
\hline & & Surface water & 2100 & (Elliott et al., 2018) \\
\hline & Vietnam & River & 230 & (Chau et al., 2018) \\
\hline \multirow[t]{5}{*}{ Lincomycin } & China & River & 10.1 & (He et al., 2018) \\
\hline & & Groundwater & 339 & (Yang et al., 2018) \\
\hline & & Surface water & 2840 & (Yang et al., 2018) \\
\hline & Korea & Coastal area & 438 & (Kim et al., 2017) \\
\hline & Taiwan & Aquaculture ponds & $2.9-226$ & (Lai et al., 2018) \\
\hline Lorazepam & China & Surface water & $3.21-8.27$ & (Xiang et al., 2018) \\
\hline \multirow[t]{5}{*}{ Losartan } & Brazil & Seawater & $0.60-8.70$ & (Sanzi et al., 2018) \\
\hline & China & WWTP & $19.7-844$ & (Wang et al., 2018) \\
\hline & Colombia & WWTP & $761-2760$ & (Botero-coy et al., 2018) \\
\hline & France & Treatment wetlands & 22,867 & (Nuel et al., 2018) \\
\hline & Italy & River & 149 & (Mandaric et al., 2017) \\
\hline Mefenamic acid & China & Groundwater & $1.86-3.40$ & (L. Ma et al., 2018) \\
\hline Meprobamate & USA & Surface water & 110 & (Elliott et al., 2018) \\
\hline Metamizole & Spain & WWTP & 3810 & (Afonso-Olivares et al., 2017) \\
\hline \multirow[t]{4}{*}{ Metformin } & Saudi Arabia & Sea water & 4801 & (Ali et al., 2017) \\
\hline & United Kingdom & River & $45.2-2595$ & (Burns et al., 2018) \\
\hline & USA & Surface water & 34,000 & (Elliott et al., 2018) \\
\hline & Vietnam & River & 8250 & (Chau et al., 2018) \\
\hline Methocarbamol & USA & Surface water & 590 & (Elliott et al., 2018) \\
\hline \multirow[t]{7}{*}{ Metoprolol } & China & WWTP & $16.1-1372.8$ & (Zhang et al., 2018) \\
\hline & & WWTP & $2.26-400$ & (Wang et al., 2018) \\
\hline & & Surface water & 130 & (Yao et al., 2018) \\
\hline & & Landfill leachates & $5390-14,100$ & (Sui et al., 2017) \\
\hline & France & Treatment wetlands & 890 & (Nuel et al., 2018) \\
\hline & Italy & River & 57.7 & (Mandaric et al., 2017) \\
\hline & USA & Surface water & 410 & (Elliott et al., 2018) \\
\hline Metoprolol acid & China & Surface water & $7.6-324$ & (Peng et al., 2018) \\
\hline \multirow[t]{2}{*}{ Metronidazole } & China & Surface water & 190 & (Yao et al., 2018) \\
\hline & Italy & River & 171 & (Mandaric et al., 2017) \\
\hline Mianserin & China & Surface water & $0.11-0.52$ & (Xiang et al., 2018) \\
\hline Miconazole & China & Groundwater & 2.56 & (Yang et al., 2018) \\
\hline \multirow[t]{2}{*}{$\mathrm{N}, \mathrm{N}$-diethyl-meta toluamide } & China & WWTP & $22.6-469$ & (Zhang et al., 2018) \\
\hline & & Groundwater & $9.20-15.8$ & (L. Ma et al., 2018) \\
\hline $\mathrm{N}-$ Acetyl-4-amino antipyrine & China & Surface water & $25-213$ & (Peng et al., 2018) \\
\hline Nalidixic acid & China & WWTP & $4.7-199.7$ & (Zhang et al., 2018) \\
\hline \multirow[t]{19}{*}{ Naproxen } & Antarctic Peninsula & Stream & 333 & (González-Alonso et al., 2017) \\
\hline & Brazil & Surface water & $6.67-145$ & (Ribeiro de Sousa et al., 2018) \\
\hline & & River & 340 & (Honjo et al., 2017) \\
\hline & China & River & 0.6 & (He et al., 2018) \\
\hline & & WWTP & 16.8 & (Wang et al., 2018) \\
\hline & & Surface water & 110 & (Yao et al., 2018) \\
\hline & & River & 10 & (Zha et al., 2017) \\
\hline & & Surface water & $4-125$ & (Peng et al., 2018) \\
\hline & Colombia & WWTP & $432-3160$ & (Botero-coy et al., 2018) \\
\hline & Czech Republic & Surface water & 1424 & (Marsik et al., 2017) \\
\hline & France & Treatment Wetlands & 19,904 & (Nuel et al., 2018) \\
\hline & Iran & WWTP & $40-1630$ & (Biel-maeso et al., 2018b) \\
\hline & Italy & River & 73.1 & (Mandaric et al., 2017) \\
\hline & Mediterranean Sea & Sea water & 1.7 & (Brumovský et al., 2017) \\
\hline & Pakistan & WWTP & 464,000 & (Ashfaq et al., 2017) \\
\hline & Slovenia & WWTP & $2.62-235$ & (Cesen et al., 2018) \\
\hline & & River & $2.98-221$ & (Cesen et al., 2018) \\
\hline & Spain & Sea water & 95.8 & (Biel-maeso et al., 2018a) \\
\hline & & WWTP & 872 & (Afonso-Olivares et al., 2017) \\
\hline \multirow[t]{2}{*}{ Nicotine } & Korea & River & $59-2040$ & (Ibe et al., 2018) \\
\hline & & WWTP & $345-3532$ & \\
\hline Nordiazepam & China & Surface water & $0.44-1.22$ & (Xiang et al., 2018) \\
\hline \multirow[t]{5}{*}{ Norfloxacin } & China & WWTP & 624 & (Wang et al., 2018) \\
\hline & Colombia & WWTP & $350-606$ & (Botero-coy et al., 2018) \\
\hline & Iran & WWTP & $5-350$ & (Biel-maeso et al., 2018b) \\
\hline & Spain & Sea water & 207.5 & (Biel-maeso et al., 2018a) \\
\hline & Vietnam & River & $45-22,319$ & (Thai et al., 2018) \\
\hline Norverapamil & Italy & River & 65.5 & (Mandaric et al., 2017) \\
\hline Ofloxacin & China & River & 23 & (Lin et al., 2018a) \\
\hline
\end{tabular}


Table 2 (Continued)

\begin{tabular}{|c|c|c|c|c|}
\hline Contaminant & Location & Source & Concentration (ng/L) & Reference \\
\hline & & River & 8 & (He et al., 2018) \\
\hline & & WWTP & $2.88-384$ & (Wang et al., 2018) \\
\hline & & Groundwater & 6.83 & (Yang et al., 2018) \\
\hline & & Surface water & 43.5 & (Yang et al., 2018) \\
\hline & & WWTP & 46 & (Lin et al., 2018b) \\
\hline & France & Treatment wetlands & 387 & (Nuel et al., 2018) \\
\hline & Iran & WWTP & $37-1470$ & (Biel-maeso et al., 2018b) \\
\hline & Korea & Coastal area & 12.4 & (Kim et al., 2017) \\
\hline & Pakistan & WWTP & 81,000 & (Ashfaq et al., 2017) \\
\hline & Spain & Sea water & 34.4 & (Biel-maeso et al., 2018a) \\
\hline & & Groundwater & 18.15 & (Boy-roura et al., 2018) \\
\hline & Vietnam & River & $23-85,190$ & (Thai et al., 2018) \\
\hline Omeprazole & France & Treatment wetlands & 4979 & (Nuel et al., 2018) \\
\hline \multirow[t]{2}{*}{ Oxazepam } & China & Surface water & $4.95-7.96$ & (Xiang et al., 2018) \\
\hline & France & Seawater & 1.74 & (Poi et al., 2018) \\
\hline Oxcarbazepine & France & Treatment wetlands & 13,727 & (Nuel et al., 2018) \\
\hline \multirow[t]{3}{*}{ Oxytetracycline } & China & Surface water & 230 & (Yao et al., 2018) \\
\hline & China & Surface water & 1880 & (Yang et al., 2018) \\
\hline & Taiwan & Aquaculture ponds & 75 & (Lai et al., 2018) \\
\hline \multirow[t]{8}{*}{ Paracetamol } & China & River & 16 & (Lin et al., 2018a) \\
\hline & & Surface water & 17.8 & (Yang et al., 2018) \\
\hline & France & Treatment wetlands & 19,810 & (Nuel et al., 2018) \\
\hline & India & River & 1565 & (Mutiyar et al., 2018) \\
\hline & Mediterranean Sea & Marine water & $0.468-1.70$ & (Brumovský et al., 2017) \\
\hline & Pakistan & WWTP & 64,000 & (Ashfaq et al., 2017) \\
\hline & Portugal & River & 69.15 & (Pereira et al., 2017) \\
\hline & United Kingdom & River & $14.3-9822$ & (Burns et al., 2018) \\
\hline Paraxanthine & Spain & WWTP & $999>0$ & (Afonso-Olivares et al., 2017) \\
\hline Paroxetine & China & River & 2.1 & (Wu et al., 2017) \\
\hline Phenacetin & China & River & 296 & (Zha et al., 2017) \\
\hline \multirow[t]{3}{*}{ Phenazone } & China & Surface water & $2.12-66.40$ & (Peng et al., 2018) \\
\hline & Italy & River & 0.956 & (Mandaric et al., 2017) \\
\hline & Spain & Sea water & 309 & (Biel-maeso et al., 2018a) \\
\hline Piroxicam & Italy & River & 42.2 & (Mandaric et al., 2017) \\
\hline Pravastatin & Italy & River & 40.89 & (Mandaric et al., 2017) \\
\hline \multirow[t]{7}{*}{ Propranolol } & Brazil & Surface water & $5.46-48.1$ & (Ribeiro de Sousa et al., 2018) \\
\hline & China & WWTP & $1.9-17.2$ & (Zhang et al., 2018) \\
\hline & Iran & WWTP & $9-235$ & (Biel-maeso et al., 2018b) \\
\hline & Italy & River & 57 & (Mandaric et al., 2017) \\
\hline & Korea & Coastal area & 11.9 & (Kim et al., 2017) \\
\hline & Spain & Sea water & 5.9 & (Biel-maeso et al., 2018a) \\
\hline & United Kingdom & River & 64.9 & (Burns et al., 2018) \\
\hline \multirow[t]{2}{*}{ Ranitidine } & Iran & WWTP & $499-7500$ & (Biel-maeso et al., 2018b) \\
\hline & United Kingdom & River & 74 & (Burns et al., 2018) \\
\hline \multirow[t]{4}{*}{ Roxithromycin } & China & River & $1.4-190$ & (Lin et al., 2018a) \\
\hline & & WWTP & $1.9-269$ & (Zhang et al., 2018) \\
\hline & & Surface water & 480 & (Yao et al., 2018) \\
\hline & & WWTP & $110-210$ & (Lin et al., 2018b) \\
\hline \multirow[t]{6}{*}{ Salicylic acid } & Brazil & River & 5170 & (Honjo et al., 2017) \\
\hline & Germany & Surface water & $2.2-51$ & (Fisch et al., 2017) \\
\hline & Iran & WWTP & $23-419$ & (Biel-maeso et al., 2018b) \\
\hline & Italy & River & 47.8 & (Mandaric et al., 2017) \\
\hline & Spain & Sea water & 977 & (Biel-maeso et al., 2018a) \\
\hline & Vietnam & WWTP & $460-660$ & (Nguyen et al., 2018) \\
\hline Sertraline & China & River & 5 & (Wu et al., 2017) \\
\hline Sotalol & Italy & River & 49.4 & (Mandaric et al., 2017) \\
\hline Sparfloxacin & Pakistan & WWTP & 19,000 & (Ashfaq et al., 2017) \\
\hline Spiramycin & Iran & WWTP & $181-2790$ & (Biel-maeso et al., 2018b) \\
\hline \multirow[t]{6}{*}{ Sulfadiazine } & Bangladesh & River & 0.58 & (Hossain et al., 2018) \\
\hline & China & River & $0.93-68$ & (Lin et al., 2018a) \\
\hline & & WWTP & $1.22-41.03$ & (Zhang et al., 2018) \\
\hline & & WWTP & $1.8-57$ & (Lin et al., 2018b) \\
\hline & & Landfill leachates & $540-4690$ & (Sui et al., 2017) \\
\hline & Germany & Surface water & $0.9-7.6$ & (Fisch et al., 2017) \\
\hline Sulfaguanidine & Taiwan & Aquaculture ponds & 72.6 & (Lai et al., 2018) \\
\hline Sulfamerazine & Germany & Surface water & $0.7-1.2$ & (Fisch et al., 2017) \\
\hline \multirow[t]{2}{*}{ Sulfamethazine } & China & WWTP & $3.7-26$ & (Lin et al., 2018b) \\
\hline & China & Landfill leachates & $730-2390$ & (Sui et al., 2017) \\
\hline Sulfamethizole & Iran & WWTP & $11-480$ & (Biel-maeso et al., 2018b) \\
\hline
\end{tabular}


Table 2 (Continued)

\begin{tabular}{|c|c|c|c|c|}
\hline Contaminant & Location & Source & Concentration (ng/L) & Reference \\
\hline & Spain & Sea water & 67.1 & (Biel-maeso et al., 2018a) \\
\hline \multirow[t]{25}{*}{ Sulfamethoxazole } & Vietnam & River & $10-252,082$ & (Thai et al., 2018) \\
\hline & Bangladesh & River & 7.24 & (Hossain et al., 2018) \\
\hline & \multirow[t]{7}{*}{ China } & River & 17.4 & (He et al., 2018) \\
\hline & & Surface water & 380 & (Yao et al., 2018) \\
\hline & & Groundwater & 25.7 & (Yang et al., 2018) \\
\hline & & WWTP & $19-43$ & (Lin et al., 2018b) \\
\hline & & Landfill leachates & 2330 & (Sui et al., 2017) \\
\hline & & River & 320 & (Zha et al., 2017) \\
\hline & & Surface water & $2.14-57.88$ & (Peng et al., 2018) \\
\hline & \multirow[t]{2}{*}{ France } & Seawater & 1.6 & (Poi et al., 2018) \\
\hline & & Treatment wetlands & 5118 & (Nuel et al., 2018) \\
\hline & Germany & Surface water & $0.6-47.5$ & (Fisch et al., 2017) \\
\hline & USA & Lake & 1400 & (Elliott et al., 2018) \\
\hline & Hungary & Surface water & 1 & (Bókony et al., 2018) \\
\hline & Iran & WWTP & $26-633$ & (Biel-maeso et al., 2018b) \\
\hline & Italy & River & 106.7 & (Mandaric et al., 2017) \\
\hline & Korea & Coastal area & 2.2 & (Kim et al., 2017) \\
\hline & Mediterranean sea & Marine water & $0.007-0.017$ & (Brumovský et al., 2017) \\
\hline & Saudi Arabia & Sea water & 62 & (Ali et al., 2017) \\
\hline & Spain & Sea water & 99 & (Biel-maeso et al., 2018a) \\
\hline & & WWTP & 1520 & (Afonso-Olivares et al., 2017) \\
\hline & & Groundwater & $0.68-28.60$ & (Boy-roura et al., 2018) \\
\hline & Taiwan & Aquaculture ponds & $2.2-23.2$ & (Lai et al., 2018) \\
\hline & United Kingdom & River & 33 & (Burns et al., 2018) \\
\hline & USA & Surface water & 1400 & (Elliott et al., 2018) \\
\hline \multirow[t]{2}{*}{ Sulfamonomethoxine } & China & River & 7.4 & (He et al., 2018) \\
\hline & Taiwan & Aquaculture ponds & $1.5-98$ & (Lai et al., 2018) \\
\hline Sulfaquinoxaline & China & River & $4>$ & (He et al., 2018) \\
\hline Sulfathiazole & Korea & Coastal area & $7.01-18.6$ & (Kim et al., 2017) \\
\hline Temazepam & China & Surface water & $1.15-2.4$ & (Xiang et al., 2018) \\
\hline Testosterone & Hungary & Surface water & 10 & (Bókony et al., 2018) \\
\hline Tetracycline & Spain & Sea water & 63.3 & (Biel-maeso et al., 2018a) \\
\hline Traimterene & USA & Surface water & 380 & (Elliott et al., 2018) \\
\hline \multirow[t]{3}{*}{ Tramadol } & France & Treatment wetlands & 193,720 & (Nuel et al., 2018) \\
\hline & United Kingdom & River & $21-650$ & (Burns et al., 2018) \\
\hline & USA & Surface water & 860 & (Elliott et al., 2018) \\
\hline \multirow[t]{12}{*}{ Trimethoprim } & Bangladesh & River & 17.2 & (Hossain et al., 2018) \\
\hline & China & Surface water & 4500 & (Yao et al., 2018) \\
\hline & & WWTP & $6.2-15$ & (Lin et al., 2018b) \\
\hline & & Landfill leachates & $1550-6000$ & (Sui et al., 2017) \\
\hline & Germany & Surface water & $1.6-17.8$ & (Fisch et al., 2017) \\
\hline & Iran & WWTP & $33-788$ & (Biel-maeso et al., 2018b) \\
\hline & Italy & River & 196 & (Mandaric et al., 2017) \\
\hline & Korea & Coastal area & 5.3 & (Kim et al., 2017) \\
\hline & Spain & Sea water & 10.6 & (Biel-maeso et al., 2018a) \\
\hline & & WWTP & 31 & (Afonso-Olivares et al., 2017) \\
\hline & Taiwan & Aquaculture ponds & $1.5-9.4$ & (Lai et al., 2018) \\
\hline & Vietnam & River & $16-106,587$ & (Thai et al., 2018) \\
\hline Trimipramine & China & River & 2.1 & (Wu et al., 2017) \\
\hline Valsartan & Italy & River & 344 & (Mandaric et al., 2017) \\
\hline \multirow[t]{3}{*}{ Venlafaxine } & China & River & 4.1 & (Wu et al., 2017) \\
\hline & Italy & River & 197 & (Mandaric et al., 2017) \\
\hline & USA & Surface water & 320 & (Elliott et al., 2018) \\
\hline Verapamil & Italy & River & 20.81 & (Mandaric et al., 2017) \\
\hline \multicolumn{5}{|l|}{ Agricultural } \\
\hline \multirow[t]{2}{*}{ 2-Hydroxyatrazine } & Brazil & River & $19.4-72.3$ & (Sposito et al., 2018) \\
\hline & China & Surface water & $22-2680$ & (Peng et al., 2018) \\
\hline Acetamiprid & China & Surface water & $2.2-58$ & (Peng et al., 2018) \\
\hline Aldrin & Brazil & River & 6.05 & (Yamamoto et al., 2018) \\
\hline Aminomethylphosphonic acid & Hungary & Surface water & $2620-25,900$ & (Bókony et al., 2018) \\
\hline \multirow[t]{3}{*}{ Atrazine } & Brazil & River & 42.1 & (Sposito et al., 2018) \\
\hline & China & Surface water & $21.28-1726$ & (Peng et al., 2018) \\
\hline & USA & Surface water & 810 & (Elliott et al., 2018) \\
\hline Azoxystrobin & China & Surface water & $2.5-45$ & (Peng et al., 2018) \\
\hline \multirow{4}{*}{ Carbendazim } & China & Surface water & 45.8 & (Yang et al., 2018) \\
\hline & & Groundwater & 9.28 & (Yang et al., 2018) \\
\hline & & Surface water & $108-1785$ & (Peng et al., 2018) \\
\hline & Europe & WWTP & 78 & (Merel et al., 2018) \\
\hline
\end{tabular}


Table 2 (Continued)

\begin{tabular}{|c|c|c|c|c|}
\hline Contaminant & Location & Source & Concentration (ng/L) & Reference \\
\hline Dimethoate & China & Surface water & $2.1-57$ & (Peng et al., 2018) \\
\hline \multirow[t]{2}{*}{ Diuron } & Brazil & River & $6.2-11.7$ & (Sposito et al., 2018) \\
\hline & China & Surface water & $1.7-107$ & (Peng et al., 2018) \\
\hline Endosulfan & Brazil & River & 6.13 & (Yamamoto et al., 2018) \\
\hline Fipronil & Brazil & River & 29.2 & (Sposito et al., 2018) \\
\hline Glyphosate & Hungary & Surface water & $2360-15,000$ & (Bókony et al., 2018) \\
\hline Heptachlor & Brazil & River & $1.22-3.53$ & (Yamamoto et al., 2018) \\
\hline Hexazinone & Brazil & River & 12.7 & (Sposito et al., 2018) \\
\hline \multirow[t]{2}{*}{ Imidacloprid } & Brazil & River & 31.4 & (Sposito et al., 2018) \\
\hline & China & Surface water & $10.9-1886$ & (Peng et al., 2018) \\
\hline Imidacloprid urea & China & Surface water & $1.1-5238$ & (Peng et al., 2018) \\
\hline Isoproturon & China & Surface water & $3-847$ & (Peng et al., 2018) \\
\hline Malathion & Brazil & River & 50.4 & (Sposito et al., 2018) \\
\hline Metalaxyl & China & Surface water & $1-30.77$ & (Peng et al., 2018) \\
\hline \multirow{2}{*}{ Metolachlor } & USA & Surface water & 1600 & (Elliott et al., 2018) \\
\hline & China & Surface water & $9.44-316$ & (Peng et al., 2018) \\
\hline Propiconazole & China & Surface water & $1.8-810$ & (Peng et al., 2018) \\
\hline Tebuconazole & China & Surface water & $3.6-133$ & (Peng et al., 2018) \\
\hline Tebuthiuron & Brazil & River & $10.4-25$ & (Sposito et al., 2018) \\
\hline Terbuthylazine & Hungary & Surface water & 330 & (Bókony et al., 2018) \\
\hline \multirow[t]{2}{*}{ Terbutryn } & Hungary & Surface water & 30 & (Bókony et al., 2018) \\
\hline & China & Surface water & $5.9-1687$ & (Peng et al., 2018) \\
\hline Thiamethoxam & China & Surface water & $2.9-90.8$ & (Peng et al., 2018) \\
\hline \multicolumn{5}{|l|}{ Drugs } \\
\hline \multirow[t]{2}{*}{ Benzoylecgonine } & Italy & Drinking water & 0.61 & (Riva et al., 2018) \\
\hline & Australia & WWTP & 117 & (Yadav et al., 2018) \\
\hline Cocaine & Italy & Drinking water & 4.44 & (Riva et al., 2018) \\
\hline \multirow[t]{4}{*}{ Codeine } & Australia & WWTP & 1721 & (Yadav et al., 2018) \\
\hline & Croatia & WWTP & 379 & (Krizman-matasic et al., 2018) \\
\hline & & River & 4 & (Krizman-matasic et al., 2018) \\
\hline & Vietnam & WWTP & 50 & (Nguyen et al., 2018) \\
\hline Ketamine & Taiwan & Aquaculture ponds & $2.8-10.8$ & (Lai et al., 2018) \\
\hline \multirow{2}{*}{ Methadone } & Croatia & WWTP & 65 & (Krizman-matasic et al., 2018) \\
\hline & Taiwan & Aquaculture ponds & $0.3-13.7$ & (Lai et al., 2018) \\
\hline \multirow[t]{2}{*}{ Methamphetamine } & Taiwan & Aquaculture ponds & $20.5-22.7$ & (Lai et al., 2018) \\
\hline & Vietnam & WWTP & $100-180$ & (Nguyen et al., 2018) \\
\hline \multirow[t]{2}{*}{ Morphine } & Australia & WWTP & 104 & (Yadav et al., 2018) \\
\hline & Croatia & WWTP & 52 & (Krizman-matasic et al., 2018) \\
\hline \multirow[t]{2}{*}{ Nicotine } & Italy & Drinking water & 28.3 & (Riva et al., 2018) \\
\hline & USA & Surface water & 490 & (Elliott et al., 2018) \\
\hline \multicolumn{5}{|l|}{ Food industry } \\
\hline Bisphenol A & Brazil & River & $9.9-48.7$ & (Sposito et al., 2018) \\
\hline & China & River & 1131 & (Tan et al., 2018) \\
\hline & & River & $23-107$ & (Niu and Zhang, 2018) \\
\hline & & Surface water & $26-720$ & (Yanhua Liu et al., 2017) \\
\hline & & River & $12.75-62.78$ & (Diao et al., 2017) \\
\hline & & River & $1.7-563$ & (Yan-hua Liu et al., 2017) \\
\hline & Italy & Drinking water & $9.72-683$ & (Riva et al., 2018) \\
\hline & Slovenia & WWTP & $20.3-118$ & (Cesen et al., 2018) \\
\hline & Thailand & Freshwater & 50.67 & (Ocharoen et al., 2018) \\
\hline & USA & Surface water & 2700 & (Elliott et al., 2018) \\
\hline Caffeine & Brazil & River & $20-1040$ & (Sposito et al., 2018) \\
\hline & & River & $27.9-24,961$ & (Ribeiro de Sousa et al., 2018) \\
\hline & China & Surface water & 3500 & (Yao et al., 2018) \\
\hline & & Landfill leachates & $1700-349,000$ & (Sui et al., 2017) \\
\hline & & WWTP & $2.42-686$ & (Wang et al., 2018) \\
\hline & & Surface water & 767 & (Yang et al., 2018) \\
\hline & & Groundwater & 50.9 & (Yang et al., 2018) \\
\hline & Hungary & Surface water & $40-90$ & (Bókony et al., 2018) \\
\hline & India & River & 2640 & (Mutiyar et al., 2018) \\
\hline & Italy & Drinking water & $2.4-5.2$ & (Riva et al., 2018) \\
\hline & Mediterranean Sea & Marine water & $0.030-0.111$ & (Brumovský et al., 2017) \\
\hline & Saudi Arabian & Sea water & 7708 & (Ali et al., 2017) \\
\hline & Slovenya & WWTP & $58-7630$ & (Cesen et al., 2018) \\
\hline & Spain & WWTP & $166-186$ & (Biel-maeso et al., 2018a) \\
\hline & & Coastal water & $6.1-327.3$ & (Biel-maeso et al., 2018a) \\
\hline & & Oceanic water & $4.3-96.6$ & (Biel-maeso et al., 2018a) \\
\hline & Taiwan & Aquaculture ponds & $1.8-276$ & (Lai et al., 2018) \\
\hline & USA & Surface water & 6600 & (Elliott et al., 2018) \\
\hline
\end{tabular}


Table 2 (Continued)

\begin{tabular}{|c|c|c|c|c|}
\hline Contaminant & Location & Source & Concentration (ng/L) & Reference \\
\hline & Vietnam & Surface water & 13,000 & (Chau et al., 2018) \\
\hline & & WWTP & 1600 & (Nguyen et al., 2018) \\
\hline Di(2-ethylhexyl)adipate & Vietnam & Surface water & 440 & (Chau et al., 2018) \\
\hline \multirow[t]{3}{*}{ Diethyl phthalate } & China & River & $25-310$ & (Niu and Zhang, 2018) \\
\hline & Hungary & Surface water & $30-250$ & (Bókony et al., 2018) \\
\hline & Vietnam & Surface water & 7490 & (Chau et al., 2018) \\
\hline Di-n-butyl phthalate & Vietnam & Surface water & 4920 & (Chau et al., 2018) \\
\hline Triphenylphosphate & Vietnam & Surface water & 140 & (Chau et al., 2018) \\
\hline \multicolumn{5}{|l|}{ Personal Care Products } \\
\hline 2-Ethylhexyl methoxycinnamate & Australia & Surface water & $8.9-640$ & (Allinson et al., 2018) \\
\hline 2-Hydroxy-4-methoxybenzophenone & Australia & Surface water & $4.3-7.1$ & (Allinson et al., 2018) \\
\hline 2-Phenoxyethanol & Australia & Surface water & $7.6-315$ & (Allinson et al., 2018) \\
\hline \multirow[t]{4}{*}{ 4-Methylbenzilidene camphor } & Australia & Surface water & 642 & (Allinson et al., 2018) \\
\hline & China & WWTP & $0.442-57.2$ & (Wang et al., 2018) \\
\hline & Italy & WWTP & $<1.8$ & (Palmiotto et al., 2018) \\
\hline & & River & 61.65 & (Mandaric et al., 2017) \\
\hline Benzophenone & Australia & Surface water & $21.8-36.4$ & (Allinson et al., 2018) \\
\hline Benzyl salicylate & Australia & Surface water & 6 & (Allinson et al., 2018) \\
\hline Benzophenone-1 (BP1) & Germany & Surface water & $1.3-2.8$ & (Fisch et al., 2017) \\
\hline \multirow[t]{5}{*}{ Benzophenone-3 (BP3) } & China & WWTP & 8.72 & (Wang et al., 2018) \\
\hline & Germany & Surface water & $6.7-11.4$ & (Fisch et al., 2017) \\
\hline & Italy & WWTP & 4.1 & (Palmiotto et al., 2018) \\
\hline & & Drinking water & $1.1-5.7$ & (Riva et al., 2018) \\
\hline & & River & 14.3 & (Mandaric et al., 2017) \\
\hline Benzophenone-4 (BP4) & Italy & WWTP & 454.7 & (Palmiotto et al., 2018) \\
\hline Butyl paraben & China & Surface water & 0.4 & (Yang et al., 2018) \\
\hline \multirow[t]{9}{*}{ DEET } & China & Surface water & $9.9-574$ & (Peng et al., 2018) \\
\hline & & WWTP & $38.8-57.2$ & (L. Ma et al., 2018) \\
\hline & & Groundwater & $9.20-15.8$ & (L. Ma et al., 2018) \\
\hline & & Surface water & 101 & (Yang et al., 2018) \\
\hline & & Groundwater & 53.8 & (Yang et al., 2018) \\
\hline & Mediterranean Sea & Marine water & $0.506-1.21$ & (Brumovský et al., 2017) \\
\hline & Saudi Arabia & Sea water & 49 & (Ali et al., 2017) \\
\hline & USA & Surface water & 5100 & (Elliott et al., 2018) \\
\hline & Vietnam & WWTP & $300-400$ & (Nguyen et al., 2018) \\
\hline \multirow[t]{2}{*}{ Ethyl paraben } & Australia & Surface water & 245 & (Allinson et al., 2018) \\
\hline & China & WWTP & 1.9 & (W. L. Ma et al., 2018) \\
\hline Galaxolide & Australia & Surface water & 10.2 & (Allinson et al., 2018) \\
\hline НHCB & USA & Surface water & 2200 & (Elliott et al., 2018) \\
\hline Lauryl diethanolamide & China & Surface water & $6.2-646$ & (Peng et al., 2018) \\
\hline \multirow[t]{7}{*}{ Methyl paraben } & Australia & Surface water & $4-1770$ & (Allinson et al., 2018) \\
\hline & China & River & $3.2-10.3$ & (He et al., 2018) \\
\hline & & WWTP & 57.6 & (W. L. Ma et al., 2018) \\
\hline & & WWTP & 94.4 & (Wang et al., 2018) \\
\hline & & Surface water & 24.4 & (Yang et al., 2018) \\
\hline & & Groundwater & 14.9 & (Yang et al., 2018) \\
\hline & Slovenya & WWTP & $14.2-52.8$ & (Cesen et al., 2018) \\
\hline \multirow[t]{3}{*}{ Octocrylene } & Australia & Surface water & $2-109$ & (Allinson et al., 2018) \\
\hline & China & Surface water & $3-258.8$ & (Peng et al., 2018) \\
\hline & Germany & Surface water & $5.3-30.8$ & (Fisch et al., 2017) \\
\hline Octyl salycilate & Australia & Surface water & $18-31.6$ & (Allinson et al., 2018) \\
\hline Octyldimethyl PABA & Australia & Surface water & 0.4 & (Allinson et al., 2018) \\
\hline ODPABA & Italy & River & 748 & (Mandaric et al., 2017) \\
\hline \multirow{4}{*}{ PBSA } & Germany & Surface water & $1.8-836$ & (Fisch et al., 2017) \\
\hline & Italy & WWTP & 347.7 & (Palmiotto et al., 2018) \\
\hline & & Drinking water & 50 & (Riva et al., 2018) \\
\hline & & Groundwater & $3.7-1853$ & (Castiglioni et al., 2018) \\
\hline \multirow[t]{5}{*}{ Propyl paraben } & Australia & Surface water & 237 & (Allinson et al., 2018) \\
\hline & China & WWTP & 115 & (Wang et al., 2018) \\
\hline & & Surface water & 11.9 & (Yang et al., 2018) \\
\hline & & Groundwater & 9.5 & (Yang et al., 2018) \\
\hline & Slovenya & WWTP & $2.44-5.18$ & (Cesen et al., 2018) \\
\hline \multirow[t]{7}{*}{ Triclocarban } & China & Surface water & 180 & (Yao et al., 2018) \\
\hline & & WWTP & $4.78-500$ & (Wang et al., 2018) \\
\hline & & Surface water & 71.8 & (Yang et al., 2018) \\
\hline & & Groundwater & 25.8 & (Yang et al., 2018) \\
\hline & India & River & $2.2-1119$ & (Vimalkumar et al., 2018) \\
\hline & Italy & WWTP & $<0.6$ & (Palmiotto et al., 2018) \\
\hline & Mediterranean Sea & Marine water & $0.0036-0.0442$ & (Brumovský et al., 2017) \\
\hline
\end{tabular}


Table 2 (Continued)

\begin{tabular}{|c|c|c|c|c|}
\hline Contaminant & Location & Source & Concentration (ng/L) & Reference \\
\hline \multirow[t]{9}{*}{ Triclosan } & Brazil & River & $4.54-61.3$ & (Ribeiro de Sousa et al., 2018) \\
\hline & \multirow[t]{4}{*}{ China } & River & 1.5 & (He et al., 2018) \\
\hline & & WWTP & 88.8 & (Wang et al., 2018) \\
\hline & & Surface water & 105 & (Yang et al., 2018) \\
\hline & & Groundwater & 30.9 & (Yang et al., 2018) \\
\hline & \multirow[t]{2}{*}{ Italy } & WWTP & 329.7 & (Palmiotto et al., 2018) \\
\hline & & Groundwater & $32-85$ & (Castiglioni et al., 2018) \\
\hline & Mediterranean Sea & Marine water & 0.305 & (Brumovský et al., 2017) \\
\hline & Slovenia & WWTP & $5.05-12.6$ & (Cesen et al., 2018) \\
\hline Benzotriazole Ultraviolet Stabilizer-326 (UV-326) & India & River & 5.7 & (Vimalkumar et al., 2018) \\
\hline Benzotriazole Ultraviolet Stabilizer-327 (UV-327) & India & River & 9.5 & (Vimalkumar et al., 2018) \\
\hline \multirow[t]{2}{*}{ Benzotriazole Ultraviolet Stabilizer-328 (UV-328) } & Australia & Surface water & $48.4-216$ & (Allinson et al., 2018) \\
\hline & Italy & River & 669 & (Mandaric et al., 2017) \\
\hline \multirow[t]{2}{*}{ Benzotriazole Ultraviolet Stabilizer-329 (UV-329) } & India & River & 31.3 & (Vimalkumar et al., 2018) \\
\hline & Italy & River & 553 & (Mandaric et al., 2017) \\
\hline Benzotriazole Ultraviolet Stabilizer-9 (UV-9) & India & River & 28.1 & (Vimalkumar et al., 2018) \\
\hline
\end{tabular}

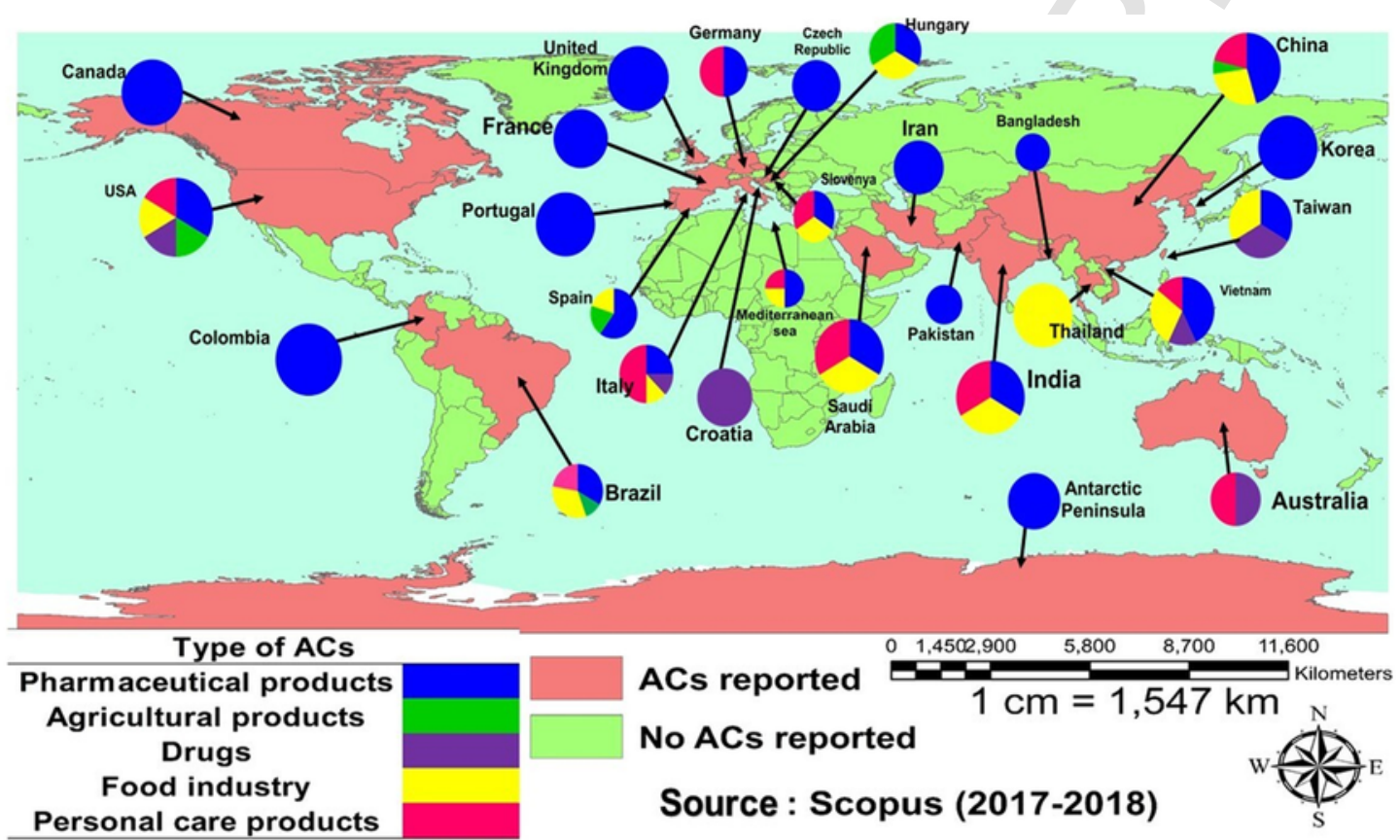

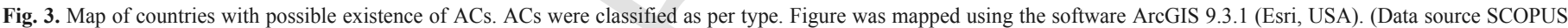
2017-2018)

ation in mRNA expression in the intestine, as well as in the expression of antioxidant genes (Stancová et al., 2015).

\subsection{Ofloxacin}

Ofloxacin is a quinolone antibiotic used for the treatment of bacterial infections and fertility treatments (Erhart et al., 1998). In Hong Kong, ofloxacin at the concentration of $0.7 \mathrm{ng} / \mathrm{L}$ has been found in the water samples from rivers (Deng et al., 2016). In Poyang Lake, the largest freshwater lake in China, ofloxacin has been detected below the quantification limit (H. Ding et al., 2017). Whereas, in the Huangpu River and Taihu Lake (Shanghai, China), ofloxacin is reported at the concentrations of approximately $28.5 \mathrm{ng} / \mathrm{L}$ and $33.6 \mathrm{ng} / \mathrm{L}$, respectively (Chen and Zhou, 2014; Xu et al., 2014). Sun et al. (2015) reported the occurrences of pharmaceutically related compounds in drinking water sources of major river watersheds in China. Whereas, the persistence of ofloxacin ranged from 4.33 to $9.43 \mathrm{ng} / \mathrm{L}$ in the surface water (Cheng et al., 2014) and $497 \mathrm{ng} / \mathrm{L}$ in WWTP (Du et al., 2017). In Shanghai, ofloxacin was found at the concentration of $2936.94 \mathrm{ng} / \mathrm{L}$ being the highest in the wastewater $(\mathrm{M} . \mathrm{H}$. Wu et al., 2016). In Pakistan, ofloxacin in wastewater was detected at a concentration of $66 \mu \mathrm{g} / \mathrm{L}$ (Ashfaq et al., 2016). Ofloxacin was also found in the range between 12 and $197 \mathrm{ng} / \mathrm{L}$ in the northern peninsula of the Antarctic (González-Alonso et al., 2017). In a karst river system (China), ofloxacin was found at a maximum concentration of $308 \mathrm{ng} /$ L (Huang et al., 2019). In aquatic ecosystems, ofloxacin at the concentrations above $10 \mathrm{mg} / \mathrm{L}$ can damage autotrophic organisms by reducing the transport of electrons from the photosystems of plant cells. Therefore, it can reduce the metabolism of plants and consequently, the rate of carbon dioxide transformation (Deng et al., 2015). In animals, the ofloxacin exposure at the concentrations of 5, 10, 20, 40, and $80 \mu \mathrm{g} / \mathrm{mL}$ is harmful. For example, in chondrocytes of juvenile rabbits, it causes an increase in oxidative stress, lipid peroxidation, DNA damage, and reduction of antioxidant enzymes ( $\mathrm{Li}$ et al., 2010). 


\subsection{Acetaminophen}

Likewise, other painkillers, Acetaminophen is an NSAID and used to control mild to moderate pain (Cao et al., 2016). In Central Europe, a study was carried out in subsurface constructed wetlands, where influent concentrations contained $>10,000 \mathrm{ng} / \mathrm{L}$ of acetaminophen, while in the effluent the overall concentration was $<50 \mathrm{ng} / \mathrm{L}$ (Chen et al., 2016). In 10 different sampling areas of the Mediterranean sea, it has been recorded in the range between 0.468 and $1.70 \mathrm{ng} / \mathrm{L}$ (Brumovský et al., 2017). In a river and a WWTP in China, acetaminophen was found at the concentration of $76.6 \mathrm{ng} / \mathrm{L}$ and $75.2 \mathrm{ng} / \mathrm{L}$, respectively $(\mathrm{He}$ et al., 2019).

The exposure to acetaminophen at the concentration of $66 \mathrm{mg} / \mathrm{kg}$ body weight (bw) has undesirable effects on living organisms, such as alterations in biochemistry and histopathology in the liver of rats (Mossa et al., 2012). It has also been demonstrated that with 5 and $15 \mathrm{mg} / \mathrm{kg}$ bw exposure to acetaminophen in the early stages of development affects the neurotransmission associated with the medulla oblongata (Blecharz-Klin et al., 2015a), or can directly affect the spinal cord (Blecharz-Klin et al., 2015b). Also, in rats, acetaminophen at 10 and $50 \mathrm{mg} / \mathrm{kg}$ bw causes the reduced synthesis of amino acids in brain cells (Blecharz-Klin et al., 2014). At concentrations of 5 and $15 \mathrm{mg} / \mathrm{kg}$ bw of acetaminophen damage the cerebellum of developing rats (Blecharz-Klin et al., 2016). While exposure to 66 and $100 \mathrm{mg}$ / $\mathrm{kg}$ bw decrease in the quantity and quality of sperm (Abedi et al., 2017). In Rhamdia quelen exposure to acetaminophen at the concentration of $0.25 \mu \mathrm{g} / \mathrm{L}$ causes a reduction in the levels of hemoglobin, hematocrit, and testosterone. In addition, it also causes hepatotoxicity and disruption in the hypothalamic-pituitary-gonadal axis (Guiloski et al., 2017a). In plants like wheat (Triticum aestivum L.), acetaminophen causes growth inhibition at $200 \mathrm{mg} / \mathrm{L}$, reduces the accumulation of chlorophyll and the synthesis of soluble proteins at 1.4 to $22.4 \mathrm{mg} / \mathrm{L}$ and 11.2 to $22.4 \mathrm{mg} / \mathrm{L}$, respectively. Acetaminophen also induces the activity of peroxidase and superoxide dismutase at concentrations ranging from 1.4 to $22.4 \mathrm{mg} / \mathrm{L}$ and damages the antioxidant defensive system (An et al., 2009). In the saltwater clam Ruditapes philippinarum, acetaminophen at $0.05 \mathrm{mg} / \mathrm{L}$ causes elevated oxidative stress, the alteration of superoxide dismutase (SOD) and reduced/oxidized glutathione (GSH/GSSG) (Correia et al., 2016).

\subsection{Progesterone}

Progesterone is a steroidal hormone involved in the female reproductive process. It can regulate the activity of a reproductive system, thus used for in vitro fertilization treatments (Dante et al., 2013). Its excessive consumption ultimately finds the way to water bodies through different routes. For instance, the activated sludge from a WWTPs in China contained progesterone in the range of $0.9-237 \mathrm{ng} /$ g (Q. Wu et al., 2016). In an earlier study, Liu et al. (2015) reported the presence of $0.47 \mathrm{ng} / \mathrm{L}$ of progesterone in the South China Sea. In France, progesterone ranging from a few $\mathrm{ng} / \mathrm{L}$ to $199 \mathrm{ng} / \mathrm{L}$ was found in $75 \%$ of the water samples collected from the Rhône-Alpes region (Vulliet and Cren-Olive, 2011). Several studies have been conducted to determine the effects caused by steroid hormones. For example, in mosquitofish Gambusia affinis, exposure for 42 days to small doses of progesterone $(4-410 \mathrm{ng} / \mathrm{L})$ caused the masculinization of female fish, reduced the fertility of females, altered the transcription of genes related to reproduction, detoxification of the liver, and alterations in ovaries, liver and gills (Hou et al., 2017). In the pond snail, Lymnaea stagnalis, progesterone at $10 \mathrm{ng} / \mathrm{L}$ changes the quantity and quality of fertilized and viable eggs (Zrinyi et al., 2017), affecting the reproduction rates of the species.

\section{Pollutants from agricultural products}

\subsection{Atrazine}

Atrazine is an herbicide which is used to kill weeds in various crops, such as sugar cane, corn, pineapple, and sorghum, among others (Wirbisky and Freeman, 2017). Because it is soluble in water, it can reach to surface and groundwater bodies by surface runoff, underground runoff, infiltration and/or accidental spillage during improper handling (EPA, 2017a). Due to its potential to filtrate through the soil, this compound has been found in drinking water reserves at the concentrations of $0.42 \mathrm{ppb}$ (Stayner et al., 2017). Exposure to $30 \mu \mathrm{g} / \mathrm{L}$ of atrazine had negative effects in the zebrafish (Danio rerio, wild-type $A B$ strain). It can cause the loss of methylation in the DNA and lead to the loss of genome protection (Wirbisky-Hershberger et al., 2017). Exposure to $10 \mathrm{mg} / \mathrm{L}$ of atrazine can changes the number of copies of some genes, as well as alterations of gene expression (Wirbisky and Freeman, 2017). It has also been proven that exposure to atrazine $(300 \mathrm{ng} / \mathrm{L})$ increases the probability of cancer, angiogenesis, and neuronal alterations (Wirbisky et al., 2016). In the crayfish Cherax quadricarinatus, the exposure to $2.5 \mathrm{mg} / \mathrm{L}$ of atrazine in the juvenile stage causes an imbalance in the sexual ratios by increasing the female proportions, which affects the reproductive rates of the species (Mac Loughlin et al., 2016).

\subsection{Carbendazim}

Carbendazim is a broad-spectrum fungicide used for pests control in agriculture (Andrade et al., 2016). In China, the maximum dietary exposure of carbendazim $(0.26 \mathrm{mg} /$ person/day $)$ through consumption of the residues existing in tomato crops has been reported ( $\mathrm{Li}$ et al., 2016). In the water flea Daphnia magna, carbendazim at 5 to $50 \mu \mathrm{g} /$ $\mathrm{L}$ had serious ecotoxicity repercussions. It causes genotoxicity, DNA damage, and reduces the rate of reproduction (Silva et al., 2015). In the zebrafish (Danio rerio), carbendazim at 20 and $100 \mu \mathrm{g} / \mathrm{L}$ induces apoptosis by up-regulation of the genes $p 53, M d m 2, B b c 3$, and Cas 8 . It is also immunotoxic and alters the endocrine system in embryonic cells (Jiang et al., 2014). The exposure of carbendazim in a range of $4-500 \mu \mathrm{g} / \mathrm{L}$ produces different trends in gene expression at larval stages (Jiang et al., 2015), as well as locomotor abnormalities and other alterations in the behavior of the species at $160 \mathrm{ng} / \mathrm{L}$ (Andrade et al., 2016). The combined effects of intra- and interspecific competition for food and exposure to carbendazim (400, 800 and $1200 \mu \mathrm{g}$ / L) were analyzed in some species (Del Arco et al., 2015). Aquatic invertebrates have a low tolerance for carbendazim. For example, in the flatworm Dugesia lugubris, $50 \%$ of the population died after $96 \mathrm{~h}$ exposure to $25 \mu \mathrm{g} / \mathrm{L}$ of carbendazim (Van Wijngaarden et al., 1998). At high concentrations $(>33 \mu \mathrm{g} / \mathrm{L})$, the organisms of the taxa Cladocera, Copepod, and Rotatoria suffered a reduction in their populations (Van Den Brink et al., 2000).

\subsection{Fipronil}

Fipronil is an insecticide used for the control of veterinary and agricultural pests (Stark and Vargas, 2005). Fipronil is leached into the environment through anthropogenic activities, such as crop spraying or the medical treatment of dogs for the control of fleas since the water used after these activities is released untreated into the environmental matrices (Teerlink et al., 2017). In the river Elbe (Germany), 
fipronil and two of its derivatives were found in a concentration of 0.5 to $1.6 \mathrm{ng} / \mathrm{L}$. The same study detected fipronil in eel's muscle $(4.05 \pm 3.73 \mathrm{ng} / \mathrm{g})$ and in liver tissue $(19.91 \pm 9.96 \mathrm{ng} / \mathrm{g})$ (Michel et al., 2016), which demonstrates the bioaccumulation in animals. In surface water from Florida (USA), fipronil has been found at the concentrations ranging from 0.5 to $207.3 \mathrm{ng} / \mathrm{L}$ (Wu et al., 2015).

In the blue crab Callinectes sapidus, fipronil in a range from 10 to $500 \mathrm{ng} / \mathrm{L}$ alter the gene expression, such as decrease of Vtg (vitellogenin) and EcR (ecdysone receptor). These effects were found to be salinity-dependent (Goff et al., 2017). In the water flea Daphnia pulex, exposure to increasing concentrations of fipronil $(0-80 \mu \mathrm{g} / \mathrm{L})$ can reduce the survival in the first, second, third and fourth stages of juvenile development (Stark and Vargas, 2005). In amphibians, like Eupemphix nattereri tadpoles, fipronil $(35-180 \mu \mathrm{g} / \mathrm{kg}$ in water and sediment) increase the oxidative stress and lipid peroxidation (Gripp et al., 2017). The exposure up to $15 \mathrm{mg} / \mathrm{kg} /$ day of fipronil can alter the cytochrome P450 enzymatic activity and liver damage in rats (Caballero et al., 2015). In the Japanese rice fish (medaka) Oryzias latipes, fipronil $(0.1$ to $910 \mu \mathrm{g} / \mathrm{L})$ caused sub-lethal alterations in embryos, such as tail deformities and reduced hatching (Wagner et al., 2017). In the Caspian white fish (Rutilus frisii), fipronil $(750 \mathrm{mg} / \mathrm{kg}$ bw intraperitoneal route) caused acute toxicity along with histopathological alterations to specific organs (Ardeshir et al., 2017).

\section{Contaminants from narcotics and other drugs}

Narcotics are used to induce human reactions like the stimulation of the central nervous system, analgesia, and narcosis (Fig. 4). The use of this kind of substances is usually regulated by governments due to their adverse human-health related effects (Argoff et al., 2009).

\subsection{Amphetamines}

Amphetamines are substances that stimulate the central nervous system. They are sympathomimetic type amines, and their mechanism of action involves the physiological pathways of several neurotransmitters, including dopamine, serotonin, and adrenaline. The effects in the human body of the consumption of these substances include an increase in blood pressure, increase in heart rate, the sensation of alertness, high stimulation, improvement of intellectual performance, and feelings of great amounts of energy, accompanied by the decrease in fatigue, sleep, and hunger. Amphetamines can cause dependence. The medical use of amphetamines is for the treatment of narcolepsy and attention deficit in children, at a recommended dose (Robledo, 2008). The existence of amphetamines in different environmental matrices, including WWTPs confirms their anthropogenic origin. The possible accumulation of $4.7 \mathrm{ng} / \mathrm{g}$ of amphetamine was recorded in aquatic species, specifically in freshwater mussels (Lasmigona costata) from the Great River in Ontario, Canada (de Solla et al., 2016). In the Puget estuary in Washington, organisms of two species, i.e., (1) Pacific staghorn sculpin (Leptocottus armatus) and (2) Chinook salmon (Oncorhynchus tshawytscha), were collected to identify the bioaccumulated substances. The study found that these species bioaccumulate 245 and $25 \mathrm{ng} / \mathrm{g}$ of amphetamine, respectively (Meador et al., 2017). Around $9.7 \mathrm{ng} / \mathrm{L}$ of amphetamine was found in the water, $3.3 \mathrm{ng} / \mathrm{g}$ in the sediment and $60 \mathrm{ng} / \mathrm{g}$ in mussels collected from 5 different places in the bay of San Francisco, California (Klosterhaus et al., 2013). This implies that following the bioaccumulation and passing through the food chain, it can be biomagnified. In the zebrafish (Danio rerio), amphetamine $(5$ and $10 \mathrm{mg} / \mathrm{L})$ has an effect of hypermobility of the fish and increase in erratic movements such as a change in the direction of movements, as well as an increase in freezing bouts (Kyzar et al., 2013).<smiles>CCCCCCCCC(C)N</smiles>

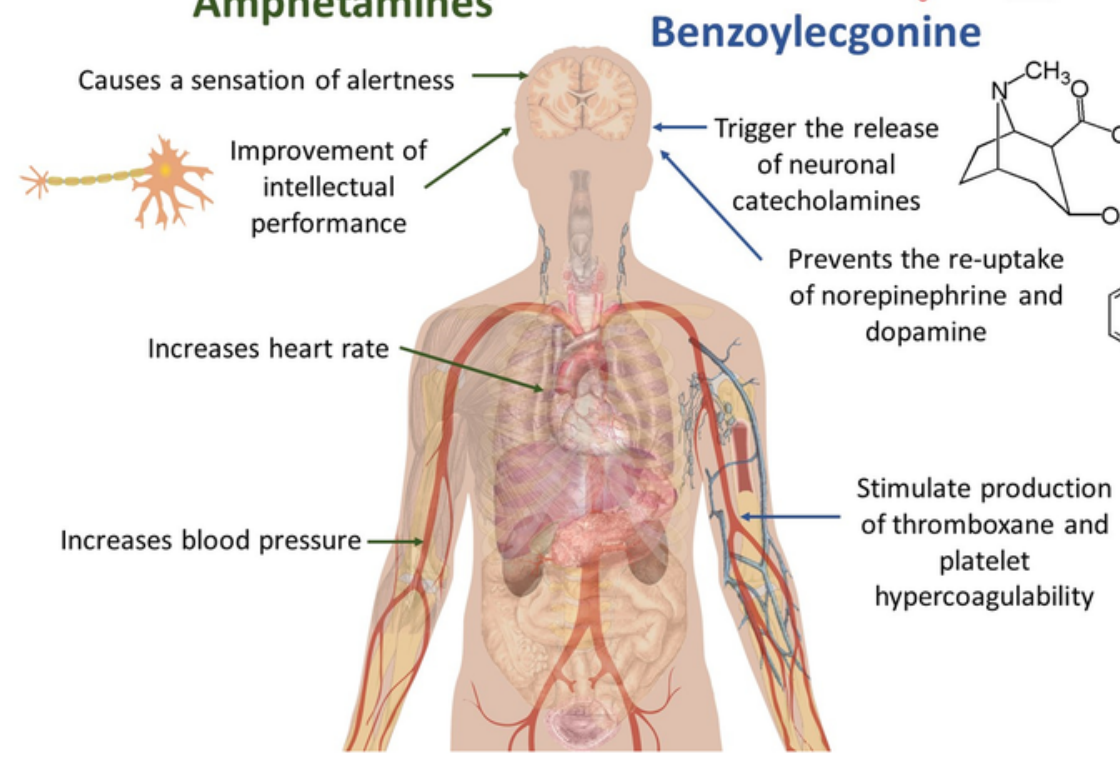

Fig. 4. Effects of amphetamines, cocaine and bencoylecgonine on the human body. 


\subsection{Cocaine}

Cocaine, an illegal substance, was found in surface water and wastewater treatment plants in Belgium, which leads to the direct exposure to animals and plants (García-Cambero et al., 2015; van Nuijs et al., 2009a). Around 28 rivers and 37 WWTPs have been found contaminated with cocaine concentrations ranging from $<1$ to $753 \mathrm{ng} / \mathrm{L}$. Those concentrations were used to estimate the amount of cocaine abuse by the population $>$. These were correlated to $>1.8 \mathrm{~g} /$ day of cocaine for every 1000 people (van Nuijs et al., 2009a). Exposure to cocaine $(0.3 \mu \mathrm{g} / \mathrm{L})$ and its metabolites changes the protein profile, alter the transport of lipids and stress response in zebrafish embryos (Parolini et al., 2017a). In Dreissena polymorpha, cocaine (40 ng/L) causes DNA damage, reduces the stability of the lysosomal membrane, increases the number of micronucleated cells, and cellular apoptosis (Binelli et al., 2012). The consumption or exposure to cocaine $(20 \mathrm{mg} / \mathrm{kg}$ bw $)$ can modify the lipid profile, the main regulators of the neuronal structure and function, of the brain in mice (Lin et al., 2017).

\subsection{Benzoylecgonine}

Benzoylecgonine is the main metabolite of cocaine and an analgesic from the pharmaceutical industry (Efeoglu et al., 2013). Several studies have been reported the existence of Benzoylecgonine in different water bodies, around the globe. For example, around 10 to $1019 \mathrm{ng} / \mathrm{L}$ in the surface waters in Brazil (Campestrini and Jardim, 2017), around 37 to $2130 \mathrm{ng} / \mathrm{L}$ in 30 Belgian WWTPs (van Nuijs et al., 2009b), and $14.7 \mathrm{ng} / \mathrm{L}$ of benzoylecgonine in 3 London rivers (Wilkinson et al., 2017). In the species, Daphnia magna, exposure to benzoylecgonine $(500 \mathrm{ng} / \mathrm{L}$ and $1000 \mathrm{ng} / \mathrm{L})$, induces oxidative stress and inhibits acetylcholine transferase. This is related to the swimming pattern and reproductive capacity of the species (Parolini et al., $2017 \mathrm{~b})$. In riparian plants and irrigated crops, this contaminant at a concentration of $1 \mathrm{ng} / \mathrm{L}$ alters the mitochondrial activity along with a reduction in the germination (García-Cambero et al., 2015). Benzoylecgonine can be transmitted from pregnant rats to their fetuses through maternal blood. Benzoylecgonine was administered to animals by an intravenous bolus dose of $1 \mathrm{mg} / \mathrm{kg}$ bw, followed by an infusion at a rate of $0.2 \mathrm{mg} / \mathrm{kg}$ bw/min, showing that exposure can cause an organism to pass it on to their offspring (Morishima et al., 2001). In Dreissena polymorpha, it was determined that exposure to benzoylecgonine (500 and $1000 \mathrm{ng} / \mathrm{L})$ affects the stability of the lysosomal membrane and imbalances the defense enzyme activity, which implies an increase of oxidative stress (Parolini et al., 2013).

\section{Contaminants from the food industry}

Caffeine is the main component of coffee, energy drinks, and some medications used for chronic diseases (Gracia-Lor et al., 2017). The caffeine found in the environment is due to anthropogenic activities (Valenzuela, 2010). In the sea water of the coast of Spain, the existence of caffeine was reported at the concentrations of $857 \mathrm{ng} / \mathrm{L}$ (Dafouz et al., 2018). A study of ten WWTPs across Europe found that the amount of caffeine discharged due to consumption by humans was between 37 and $320 \mathrm{mg} /$ person/day (Gracia-Lor et al., 2017). Exposure to caffeine has repercussions on the health of living organisms. Exposure to caffeine $(19.41 \mathrm{mg} / \mathrm{L})$ at the larval stage of Galleria mellonella affects the behavior and development, as well as increase the abundance of peptides associated with brain trauma (Maguire et al., 2017). In mice, it was determined that exposure to caffeine at the concentration of $2 \mathrm{mg} / 100 \mathrm{~g}$ bw affects embryonic de- velopment by causing a minor malformation of the phalanges of the developing limbs (Lashein et al., 2016). In zebrafish, exposure to $48.54 \mu \mathrm{g} / \mathrm{L}$ causes cellular damage, increases apoptosis, mitochondrial damage, and morphological abnormalities in the early stages of development (Rah et al., 2017).

Bisphenol A (BPA) is a chemical used in the plastic and epoxy resin industry (Staniszewska et al., 2015; Bilal et al., 2019a). BPA is used to manufacture the food packaging material, baby toys, plastic wares, compact discs, and medical instruments. The way that humans are exposed to this pollutant is through the consumption of food that has been stored in a container with BPA (NIH, 2017). In Taihu Lake, China, the concentrations of BPA and eight analogs in surface waters ranged from 49.7 to $3480 \mathrm{ng} / \mathrm{L}$. It has been found that BPA can accumulate through the food chain (Q. Wang et al., 2017). In India, BPA was found in surface water at the concentrations from 54 to $1950 \mathrm{ng} / \mathrm{L}$ (Yamazaki et al., 2015), whereas, in the Baltic region, it was found in a range of $<5.0$ to $277 \mathrm{ng} / \mathrm{L}$ in rivers and the coastal zone (Staniszewska et al., 2015). The consequences of exposure to BPA (5, 25 and $125 \mu \mathrm{g} / \mathrm{kg}$ bw) in rats include a significant decrease in daily weight gain, an increase in gamma globulin, induced damage in the liver, and promotion of death of liver cells (Kazemi et al., 2017). BPA, at concentrations of $8.6 \mathrm{mg} / \mathrm{L}$ in water and at $13.5 \mathrm{mg} / \mathrm{L}$ in sediment, in Asellus aquaticus acts as an endocrine disruptor (Plahuta et al., 2015). In rats, the exposure to BPA $(10 \mathrm{mg} / \mathrm{kg}$ bw) caused female rats to reach puberty at a younger age with a possible effect on the reproductive functions (Shi et al., 2017).

\section{Personal care products}

\subsection{Triclosan}

Triclosan is an antimicrobial present in the formulations of a large number of personal care products, such as antibacterial gels and toothpaste (Z. Wang et al., 2017). In the USA, the use of triclosan in personal care products is regulated by the FDA (EPA, 2017b). Triclosan has been found in some sediments of rivers in China (0.10-64.9 ng/ g) (Z. F. Chen et al., 2018). In Minnesota, USA, it was found in surface water $(0.005-0.31 \mu \mathrm{g} / \mathrm{L})$, WWTPs $(0.13-2.90 \mu \mathrm{g} / \mathrm{L})$ and in surface sediments $(0.9-672 \mu \mathrm{g} / \mathrm{L})$ (Lyndall et al., 2017). The effects in organisms exposed to $580 \mathrm{ng} / \mathrm{L}$ of triclosan include the increase of oxidative stress in the species Dreissena polymorpha (Riva et al., 2012). Also, triclosan reduces the lifespan, survival rate, and fecundity in Brachionus havanaensis and Plationus patulus at a concentration of $6.25 \mu \mathrm{g} / \mathrm{L}$ (González-Pérez et al., 2018). In the frog Pelophylax nigromaculatus it can disrupt gonadal differentiation and development, affecting the sex ratios of the species at a concentration of $0.868 \mu \mathrm{g} / \mathrm{L}(\mathrm{J}$. Chen et al., 2018). In a study, triclosan was administrated to mice at 10,100 and $200 \mathrm{mg} / \mathrm{kg}$ diet/day, inducing tumors in mice by involving receptors CAR and PPAR $\alpha$, which mediate the process that increases the synthesis of DNA in the liver (Z. Wang et al., 2017).

\subsection{Surfactants}

There are different types of surfactants used in personal care products and likewise, triclosan, are regulated by the FDA (EPA, 2017b). Some of the most used are alkyl sulfates. For example, sodium lauryl ether sulfate (SLS), an anionic surfactant, is a mixture of linear primary alkyl ether sulfates (AES) used as an emulsifying agent in household cleaning products. SLS concentration is generally from $0.01 \%$ to $50 \%$ in cosmetic products and $1 \%-30 \%$ in cleaning products. However, the concentration of SLS in domestic wastewater can 
vary between 0.4 and $12 \mathrm{mg} / \mathrm{L}$ (Marks et al., 2015). One of the problems with SLS is that in WWTPs it causes a decrease in the floccus size of activated sludge. In addition, it becomes toxic to microorganisms by binding to enzymes, structural proteins, and phospholipids, or by changing the hydrophobicity of the bacterial cell (Paulo et al., 2017). Studies show that different concentrations of SLS may affect aquatic organisms, for example, a concentration of $4.68 \mathrm{mg} / \mathrm{L}$ causes growth inhibition of marine microalga Dunaliella salina (Sibila et al., 2008), and $2.10 \mathrm{mg} / \mathrm{L}$ inhibits the freshwater microalgae Pseudokirchneriella subcapitata (Pavlić et al., 2005).

\section{Human consumption of anthropogenic contaminants and their presence in drinking water reservoirs}

ACs have been found in drinking water reservoirs, WWTP, DWTP, and in places where humans are in contact with these contaminated water resources (Fawell and Nieuwenhuijsen, 2003). For example, water from some rivers is used as drinking water, such as the Yangtze River in China. In this river, triclosan was found at the concentrations of $1.85 \mathrm{ng} / \mathrm{L}$, and it was estimated that the daily intake of triclosan is of $0.03 \mathrm{ng} / \mathrm{kg}$ bw/day in children and $0.02 \mathrm{ng} / \mathrm{kg}$ bw $/$ day in teenagers and adults (X. Ma et al., 2018). In Vietnam, drinking water also contains some pesticides such as butachlor $(0.47 \mu \mathrm{g} / \mathrm{L})$ and fipronil $(0.04 \mu \mathrm{g} / \mathrm{L})$. The source of contamination by pesticides may be by direct contact with the pesticide or by infiltration (Toan et al., 2013). As in the case of groundwater from the River Ganges Basin (India), several ACs have been detected, including acetaminophen, at the concentration of $1.92 \mathrm{ng} / \mathrm{L}$, caffeine at $208 \mathrm{ng} / \mathrm{L}$, carbamazepine at $27.2 \mathrm{ng} / \mathrm{L}$, sulfamethoxazole at $3.49 \mathrm{ng} / \mathrm{L}$, diclofenac at $1.56 \mathrm{ng} / \mathrm{L}$, naproxen at $2.37 \mathrm{ng} / \mathrm{L}$, ibuprofen at $49.4 \mathrm{ng} / \mathrm{L}$, and triclosan at $10.2 \mathrm{ng} /$ L (Sharma et al., 2019). In Milan, groundwater was analyzed after drinking water treatment, and the analysis profile revealed $10.3 \mathrm{ng} /$ $\mathrm{L}$ of carbamazepine, $0.61 \mathrm{ng} / \mathrm{L}$ of benzoylecgonine, $4.44 \mathrm{ng} / \mathrm{L}$ of cocaine, $683 \mathrm{ng} / \mathrm{L}$ of BPA and $5.2 \mathrm{ng} / \mathrm{L}$ of caffeine (Riva et al., 2018). Additional studies are included in Table 2.

Groundwater in Sub-Saharan Africa was reported to contain $335 \mathrm{ng} / \mathrm{L}$ of carbamazepine, $276 \mathrm{ng} / \mathrm{L}$ of ibuprofen, $518 \mathrm{ng} / \mathrm{L}$ of diclofenac, $111 \mathrm{ng} / \mathrm{L}$ of acetaminophen and $1285 \mathrm{ng} / \mathrm{L}$ of sulfamethoxazole (Branchet et al., 2019). A lake in Brazil (Guarapiranga), contained $179 \mathrm{ng} / \mathrm{L}$ of benzoylecgonine and $12 \mathrm{ng} / \mathrm{L}$ of cocaine. Also, in Brazil, drinking water from rivers that supply five cities contain $652 \mathrm{ng} / \mathrm{L}$ of benzoylecgonine and $22 \mathrm{ng} / \mathrm{L}$ of cocaine (Campestrini and Jardim, 2017). In China, two recent studies found the following ACs before and after the water treatment process in a DWTP (expressed as $\mathrm{AC}$ maximum concentration in raw water to $\mathrm{AC}$ concentration in effluent): 37.1 to $6.4 \mathrm{ng} / \mathrm{L}$ of acetaminophen, 1.01 to $0.65 \mathrm{ng} / \mathrm{L}$ of carbamazepine, 14.2 to $3.8 \mathrm{ng} / \mathrm{L}$ of caffeine, 12.0 to $5 \mathrm{ng} / \mathrm{L}$ of indomethacin, 4.3 to $2.5 \mathrm{ng} / \mathrm{L}$ of lincomycin, 35.4 to $5.4 \mathrm{ng} / \mathrm{L}$ of sulfamethoxazole, 17.0 to $3.7 \mathrm{ng} / \mathrm{L}$ of trimethoprim (Lin et al., 2016), and 34.9 to $6.5 \mathrm{ng} /$ L of BPA (Zhang et al., 2019). In a DWTP in Taiwan, BPA was found at the concentration of $38 \mathrm{ng} / \mathrm{L}$ after water treatment. Based on these results, it was estimated that the daily intake of BPA per person is between 4.3 and $76 \mathrm{ng} / \mathrm{day}$, considering that a person consumes 21 of water daily (Chen et al., 2013). Water samples from DWTP in Madrid (Spain) were analyzed and found contaminated with ACs, such as methylparaben (9.87-85.89 ng/L), ethylparaben (11.97 ng/L) and BPA $(5123 \mathrm{ng} / \mathrm{L})($ Alda et al., 2018). Another study carried out in a DWTP that treats water from the Mediterranean Llobregat River (Spain). Acetaminophen, carbamazepine, hydrochlorothiazide, thiabendazole, diltiazem, norverapamil, BPA, and propyl-paraben were detected even after the treatment process (Gabarrón et al., 2016). The ACs removal in treated water from a
WTP in the area of Gdańsk (Poland) was determined, and the results are expressed as concentration range and \% of compound removal from untreated water): $4.9-5.6 \mathrm{ng} / \mathrm{L}(0.0 \%)$ of ranitidine, $9.3-44.0 \mathrm{ng} /$ $\mathrm{L}(44.5 \%)$ of acetaminophen, $12.7-158.7 \mathrm{ng} / \mathrm{L}(61.3 \%)$ of caffeine, $2.1-6.0 \mathrm{ng} / \mathrm{L}(88.9 \%)$ of carbamazepine, $114.3 \mathrm{ng} / \mathrm{L}(-298.8 \%)$ of diclofenac and 5.7-223.6ng/L (21.2\%) of ibuprofen (Kot-Wasik et al., 2016). Drinking water samples obtained from a local water supply system in Brazil were analyzed (Sodré et al., 2018). The samples were found to contain $3.3 \mathrm{ng} / \mathrm{L}$ of atrazine and $16 \mathrm{ng} / \mathrm{L}$ of caffeine (Sodré et al., 2018). In Croatia, drinking water samples obtained from municipal water supplies contained 5-68 ng/L of atrazine (Fingler et al., 2017). In Ohio, USA, the atrazine concentration in drinking water was monitored (2006-2008), and it was detected in a range of $0-15.7 \mu \mathrm{g} / \mathrm{L}$ (Almberg et al., 2018).

Furthermore, commercial bottled waters from France and other European countries contain several ACs, i.e., diclofenac, sulfamethoxazole, carbamazepine, ofloxacin, ibuprofen, acetaminophen, caffeine, metformin $(12 \mathrm{ng} / \mathrm{L})$, salicylic acid $(16 \mathrm{ng} / \mathrm{L})$ and gabapentine $(12 \mathrm{ng} /$ L) (Lardy-Fontan et al., 2017). In Thailand, some commercial canned carbonated drinks and plastic-bottled waters were analyzed. In both types of test samples, BPA was found at the concentrations of 51-340 and $30 \mathrm{ng} / \mathrm{L}$, respectively (Chailurkit et al., 2017). While, in Lebanon, bottled water contained $1.37 \mathrm{ng} / \mathrm{L}$ of BPA (Dhaini and Nassif, 2014). In Vietnam, it was reported that 10 brands of bottled water contained some ACs from the agricultural sector, including fenobucarb, isoprothiolane, pretilachlor, fipronil, hexaconazole and azoxystrobin (Chau et al., 2015). A study on the food diet of 50 North Carolina (USA) adults, found BPA at $0.062 \mathrm{ng} / \mathrm{mL}$ in $38 \%$ of solid food and $4 \%$ of drinking water samples. It was estimated that people are consuming up to $10.7 \mathrm{ng} / \mathrm{kg} /$ day (Morgan et al., 2018). A study carried out in China with 12 adults (25 years old), ACs detected in urine with $3.5 \mu \mathrm{g}$ of triclosan $/ \mathrm{g}$ creatinine and $2.75 \mu \mathrm{g}$ of BPA/g creatinine (Li et al., 2013).

\section{Challenges and threats}

The amount of ACs found in different water bodies alarms to consider the pollution plume that is derived from various anthropogenic activities. This is not only damaging for humans but also for many other aquatic species, alike. Although the processes of the current wastewater treatment plants can reduce the pollutant load. However, the above-discussed examples reflect that treated effluents discharged into water bodies have considerable concentrations of different ACs. Thus, more robust strategies are needed for complete removal of various types of ACs. This is even highly requisite in rural areas where there is no such wastewater disposal. The filtration process (groundwater) or storage of water in reservoirs for human consumption is carried out through the flow of water in an endorheic and exorheic basin, thus making it possible for certain ACs to return to the population due to the currents of drinking water treatments. The map proposed, shows that ACs are present at detectable levels in water bodies all over the world. Since these pollutants have been found even in the polar zones, where the production of ACs is considered minimal. For all these reasons, it is necessary to optimize and redesign the treatment of waste and drinking waters for the effective removal of ACs in a safe and eco-friendly way.

\section{Concluding summary}

In summary, the presence of ACs in different environmental matrices needs more attention, planning of mitigation strategies, and implementation of strategic measures to detect and remove effectively. 
Unless otherwise, their free movement can cause uncontrol spread throughout the environment and damage various habitats. Until recently, ACs had not been actively addressed as a major environmental concern. In addition, the data discussed above with suitable examples show that the methods used are not sufficient for the removal of ACs. Clearly, more studies are needed to effectively regulate and evaluate the number of hazardous substances found in different environmental matrices around the globe. Besides effective removal, adverse effects on different organisms should also be considered with care. Although the information obtained is of great importance, the studies so far do not reflect the magnitude of the current problem with ACs, as was shown anthropogenic contaminants occurrence around the globe in 2017 and 2018 found in water bodies give the idea that the occurrence of ACs happens around the globe. The majority of the ACs reviewed in this article are endocrine disruptors, which cause changes in behavior, cellular toxicity, genotoxicity, and alter the sex ratios in organisms. These types of contaminants are already affecting the biodiversity hotspots worldwide. Furthermore, they also affect the trophic chain at all levels through bioaccumulation and biomagnification.

\section{Declaration of Competing Interest}

The authors declare no conflict of interest.

\section{Acknowledgments}

The financial support provided by the Bioprocess Research Chair (0020209I13) at Tecnologico de Monterrey, Mexico, Consejo Nacional de Ciencia y Tecnologia (CONACYT), Mexico, and CONACYT-Innovate UK project "Phycopigments" (grant \#268792) is thankfully acknowledged. The Master Scholarship awarded by Consejo Nacional de Ciencia y Tecnologia (CONACYT), Mexico to Itzel Y. López-Pacheco (637424) and Arisbe Silva-Núñez (888365) is thankfully acknowledged.

\section{References}

Abedi, N., Nabi, A., Mangoli, E., Talebi, A.R., 2017. Short and long term effects of different doses of paracetamol on sperm parameters and DNA integrity in mice. Middle East Fertil. Soc. J. 22, 323-328. https://doi.org/10.1016/j.mefs.2017.06. 001 .

Afonso-Olivares, C., Sosa-Ferrera, Z., Santana-Rodríguez, J.J., 2017. Occurrence and environmental impact of pharmaceutical residues from conventional and natural wastewater treatment plants in Gran Canaria (Spain). Sci. Total Environ. 599-600, 934-943. https://doi.org/10.1016/j.scitotenv.2017.05.058.

Alda, D., Gil, A., Gorga, M., Valc, Y., Navas, J.M., Petrovic, M., Barcel, D., 2018. Determining the presence of chemicals with suspected endocrine activity in drinking water from the Madrid region (Spain) and assessment of their estrogenic, androgenic and thyroidal activities. Chemosphere 201, 388-398. https://doi.org/10.1016/ j.chemosphere.2018.02.099.

Ali, A.M., Thorsen, H., Alarif, W., Kallenborn, R., Al-lihaibi, S.S., 2017. Occurrence of pharmaceuticals and personal care products in ef fl uent- dominated Saudi Arabian coastal waters of the Red Sea. Chemosphere 175, 505-513. https://doi.org/10. 1016/j.chemosphere.2017.02.095.

Allinson, M., Kameda, Y., Kimura, K., Allinson, G., 2018. Occurrence and assessment of the risk of ultraviolet filters and light stabilizers in Victorian estuaries. Environ. Sci. Poll. Res. 25 (12), 12022-12033.

Almberg, K.S., Turyk, M.E., Jones, R.M., Rankin, K., Freels, S., Stayner, L.T., 2018. Atrazine contamination of drinking water and adverse birth outcomes in community water systems with elevated atrazine in Ohio, 2006-2008. Int. J. Environ. Res Public Health 15, 12-15. https://doi.org/10.3390/ijerph15091889.

An, J., Zhou, Q., Sun, F., Zhang, L., 2009. Ecotoxicological effects of paracetamol on seed germination and seedling development of wheat (Triticum aestivum L.). J. Hazard. Mater. 169, 751-757. https://doi.org/10.1016/j.jhazmat.2009.04.011.

Andrade, T.S., Henriques, J.F., Almeida, A.R., Machado, A.L., Koba, O., Giang, P.T., Soares, A.M.V.M., Domingues, I., 2016. Carbendazim exposure induces develop- mental, biochemical and behavioural disturbance in zebrafish embryos. Aquat. Toxicol. 170, 390-399. https://doi.org/10.1016/j.aquatox.2015.11.017.

André, C., Gagné, F., 2017. Cumulative effects of ibuprofen and air emersion in zebra mussels Dreissena polymorpha. Environ. Toxicol. Pharmacol. 55, 156-164. https:// doi.org/10.1016/j.etap.2017.08.016.

Ardeshir, R.A., Zolgharnein, H., Movahedinia, A., Salamat, N., Zabihi, E., 2017. Comparison of waterborne and intraperitoneal exposure to fipronil in the Caspian white fish (Rutilus frisii) on acute toxicity and histopathology. Toxicol. Reports 4 , 348-357. https://doi.org/10.1016/j.toxrep.2017.06.010.

Argoff, C.E., McCleane, G., Kanner, R., 2009. Opioid analgesics. Pain Manag. Secrets 255-261. https://doi.org/10.1016/B978-0-323-04019-8.00034-2.

Ashfaq, M., Khan, K.N., Rasool, S., Mustafa, G., Saif-Ur-Rehman, M., Nazar, M.F., Sun, Q., Yu, C.P., 2016. Occurrence and ecological risk assessment of fluoroquinolone antibiotics in hospital waste of Lahore, Pakistan. Environ. Toxicol. Pharmacol. 42, 16-22. https://doi.org/10.1016/j.etap.2015.12.015.

Ashfaq, M., Nawaz Khan, K., Saif Ur Rehman, M., Mustafa, G., Faizan Nazar, M., Sun, Q., Iqbal, J., Mulla, S.I., Yu, C.P., 2017. Ecological risk assessment of pharmaceuticals in the receiving environment of pharmaceutical wastewater in Pakistan. Ecotoxicol. Environ. Saf. 136, 31-39. https://doi.org/10.1016/j.ecoenv.2016. 10.029 .

Barrios-Estrada, C., de Jesús Rostro-Alanis, M., Muñoz-Gutiérrez, B.D., Iqbal, H.M.N., Kannan, S., Parra-Saldívar, R., 2018. Emergent contaminants: endocrine disruptors and their laccase-assisted degradation - a review. Sci. Total Environ. 612, 1516-1531. https://doi.org/10.1016/J.SCITOTENV.2017.09.013.

Barrios-Estrada, C., de Jesús Rostro-Alanis, M., Parra, A.L., Belleville, M.P., Sanchez-Marcano, J., Iqbal, H.M., Parra-Saldívar, R., 2018. Potentialities of active membranes with immobilized laccase for Bisphenol A degradation. Int. J. Biol. Macromol. 108, 837-844. https://doi.org/10.1016/j.ijbiomac.2017.10.177.

Biel-maeso, M., Baena-nogueras, R.M., Corada-fernández, C., Lara-martín, P.A., 2018. Occurrence, distribution and environmental risk of pharmaceutically active compounds (PhACs) in coastal and ocean waters from the Gulf of Cadiz (SW Spain). Sci. Total Environ. 612, 649-659. https://doi.org/10.1016/j.scitotenv.2017. 08.279 .

Biel-maeso, M., Corada-fern, C., Lara-martín, P.A., 2018. Monitoring the occurrence of pharmaceuticals in soils irrigated with reclaimed wastewater. Environ. Pollut. 235, 312-321. https://doi.org/10.1016/j.envpol.2017.12.085.

Bilal, M., Iqbal, H.M., 2019. An insight into toxicity and human-health-related adverse consequences of cosmeceuticals - a review. Sci. Total Environ. 670, 555-568. https://doi.org/10.1016/j.scitotenv.2019.03.261.

Bilal, M., Asgher, M., Parra-Saldivar, R., Hu, H., Wang, W., Zhang, X., Iqbal, H.M., 2017. Immobilized ligninolytic enzymes: an innovative and environmental responsive technology to tackle dye-based industrial pollutants-a review. Sci. Total Environ. 576, 646-659. https://doi.org/10.1016/j.scitotenv.2016.10.137.

Bilal, M., Iqbal, H.M., Barceló, D., 2019. Mitigation of bisphenol A using an array of laccase-based robust bio-catalytic cues-a review. Sci. Total Environ. 689, 160-177. https://doi.org/10.1016/j.scitotenv.2019.06.403.

Bilal, M., Rasheed, T., Nabeel, F., Iqbal, H.M., Zhao, Y., 2019. Hazardous contaminants in the environment and their laccase-assisted degradation-a review. J. Environ. Manag. 234, 253-264. https://doi.org/10.1016/j.jenvman.2019.01.001.

Bilal, M., Adeel, M., Rasheed, T., Zhao, Y., Iqbal, H.M., 2019. Emerging contaminants of high concern and their enzyme-assisted biodegradation-a review. Environ. Int. 124, 336-353. https://doi.org/10.1016/j.envint.2019.01.011.

Binelli, A., Pedriali, A., Riva, C., Parolini, M., 2012. Illicit drugs as new environmental pollutants: cyto-genotoxic effects of cocaine on the biological model Dreissena polymorpha. Chemosphere 86, 906-911. https://doi.org/10.1016/j.chemosphere. 2011.10.056

Blecharz-Klin, K., Joniec-Maciejak, I., Piechal, A., Pyrzanowska, J., Wawer, A., Widy-Tyszkiewicz, E., 2014. Paracetamol impairs the profile of amino acids in the rat brain. Environ. Toxicol. Pharmacol. 37, 95-102. https://doi.org/10.1016/j.etap. 2013.11.004.

Blecharz-Klin, K., Joniec-Maciejak, I., Jawna, K., Pyrzanowska, J., Piechal, A., Wawer, A., Widy-Tyszkiewicz, E., 2015. Developmental exposure to paracetamol causes biochemical alterations in medulla oblongata. Environ. Toxicol. Pharmacol. 40, 369-374. https://doi.org/10.1016/j.etap.2015.07.001.

Blecharz-Klin, K., Joniec-Maciejak, I., Jawna, K., Pyrzanowska, J., Piechal, A., Wawer, A., Widy-Tyszkiewicz, E., 2015. Effect of prenatal and early life paracetamol exposure on the level of neurotransmitters in rats-focus on the spinal cord. Int. J. Dev. Neurosci. 47, 133-139. https://doi.org/10.1016/j.ijdevneu.2015.09.002.

Blecharz-Klin, K., Joniec-Maciejak, I., Jawna-Zboińska, K., Pyrzanowska, J., Piechal, A., Wawer, A., Widy-Tyszkiewicz, E., 2016. Cerebellar level of neurotransmitters in rats exposed to paracetamol during development. Pharmacol. Reports 68, 1159-1164. https://doi.org/10.1016/j.pharep.2016.06.005.

Blowes, D.W., Ptacek, C.J., Jambor, J.L., Weisener, C.G., 2003. The geochemistry of acid mine drainage. In: Treatise on Geochemistry. pp. 149-204. https://doi.org/10. 1016/B0-08-043751-6/09137-4.

Bókony, V., Üveges, B., Ujhegyi, N., Verebélyi, V., Nemesházi, E., Csíkvári, O., Hettyey, A., 2018. Endocrine disruptors in breeding ponds and reproductive health of 
toads in agricultural, urban and natural landscapes. Sci. Total Environ. 634, 1335-1345. https://doi.org/10.1016/j.scitotenv.2018.03.363.

Botero-coy, A.M., Martínez-pachón, D., Boix, C., Rincón, R.J., Castillo, N., Arias-marín, L.P., 2018. An investigation into the occurrence and removal of pharmaceuticals in Colombian wastewater. Sci. Total Environ. 642, 842-853. https:// doi.org/10.1016/j.scitotenv.2018.06.088.

Boy-roura, M., Mas-pla, J., Petrovic, M., Gros, M., Soler, D., Brusi, D., Menció, A., 2018. Towards the understanding of antibiotic occurrence and transport in groundwater: findings from the Baix Fluvià alluvial aquifer (NE Catalonia, Spain). Sci. Total Environ. 612, 1387-1406. https://doi.org/10.1016/j.scitotenv.2017.09.012.

Branchet, P., Ariza Castro, N., Fenet, H., Gomez, E., Courant, F., Sebag, D., Gardon, J., Jourdan, C., Ngounou Ngatcha, B., Kengne, I., Cadot, E., Gonzalez, C., 2019 Anthropic impacts on Sub-Saharan urban water resources through their pharmaceutical contamination (Yaoundé, Center Region, Cameroon). Sci. Total Environ. 660, 886-898. https://doi.org/10.1016/j.scitotenv.2018.12.256.

Brown, A.K., Wong, C.S., 2018. Distribution and fate of pharmaceuticals and their metabolite conjugates in a municipal wastewater treatment plant. Water Res. 144, 774-783. https://doi.org/10.1016/j.watres.2018.08.034

Brumovský, M., Bečanová, J., Kohoutek, J., Borghini, M., Nizzetto, L., 2017. Contaminants of emerging concern in the open sea waters of the Western Mediterranean. Environ. Pollut. 229, 976-983. https://doi.org/10.1016/j.envpol.2017.07.082.

Burns, E.E., Carter, L.J., Kolpin, D.W., Thomas-oates, J., Boxall, A.B.A., 2018. Temporal and spatial variation in pharmaceutical concentrations in an urban river system. Water Res. 137, 72-85. https://doi.org/10.1016/j.watres.2018.02.066.

Caballero, M.V., Ares, I., Martínez, M., Martínez-Larrañaga, M.R., Anadón, A., Martínez, M.A., 2015. Fipronil induces CYP isoforms in rats. Food Chem. Toxicol. 83, 215-221. https://doi.org/10.1016/j.fct.2015.06.019.

Campestrini, I., Jardim, W.F., 2017. Occurrence of cocaine and benzoylecgonine in drinking and source water in the S??o Paulo State region, Brazil. Sci. Total Environ. 576, 374-380. https://doi.org/10.1016/j.scitotenv.2016.10.089.

Cao, F., Zhang, M., Yuan, S., Feng, J., Wang, Q., Wang, W., Hu, Z., 2016. Transformation of acetaminophen during water chlorination treatment: kinetics and transformation products identification. Environ. Sci. Pollut. Res. 23, 12303-12311. https://doi.org/10.1007/s11356-016-6341-x.

Carmona, E., Andreu, V., Picó, Y., 2014. Occurrence of acidic pharmaceuticals and personal care products in Turia River Basin: from waste to drinking water. Sci. Total Environ. 484, 53-63. https://doi.org/10.1016/j.scitotenv.2014.02.085.

Castiglioni, S., Davoli, E., Riva, F., Palmiotto, M., Camporini, P., Manenti, A., Zuccato, E., 2018. Mass balance of emerging contaminants in the water cycle of a highly urbanized and industrialized area of Italy. Water Res. 131, 287-298. https:// doi.org/10.1016/j.watres.2017.12.047.

Cesen, M., Heath, D., Krivec, M., Kosmrlj, J., Kosjek, T., Heath, E., 2018. Seasonal and spatial variations in the occurrence, mass loadings and removal of compounds of emerging concern in the Slovene aqueous environment and environmental risk assessment. Environ. Pollut. J. 242, https://doi.org/10.1016/j.envpol.2018.06.052.

Chailurkit, L. or, Srijaruskul, K., Ongphiphadhanakul, B., 2017. Bisphenol A in canned carbonated drinks and plastic-bottled water from supermarkets. Expo. Heal. 9, 243-248. https://doi.org/10.1007/s12403-016-0235-5.

Chau, N.D.G., Sebesvari, Z., Amelung, W., Renaud, F.G., 2015. Pesticide pollution of multiple drinking water sources in the Mekong Delta, Vietnam: evidence from two provinces. Environ. Sci. Pollut. Res. 22, 9042-9058. https://doi.org/10.1007/ s11356-014-4034-x.

Chau, H.T.C., Kadokami, K., Duong, H.T., Kong, L., 2018. Occurrence of 1153 Organic Micropollutants in the Aquatic Environment of Vietnam. 7147-7156. https://doi.org/10.1007/s11356-015-5060-Z.

Chen, K., Zhou, J.L., 2014. Occurrence and behavior of antibiotics in water and sediments from the Huangpu River, Shanghai, China. Chemosphere 95, 604-612. https://doi.org/10.1016/j.chemosphere.2013.09.119.

Chen, H.W., Liang, C.H., Wu, Z.M., Chang, E.E., Lin, T.F., Chiang, P.C., Wang, G.S., 2013. Occurrence and assessment of treatment efficiency of nonylphenol, octylphenol and bisphenol-A in drinking water in Taiwan. Sci. Total Environ. 449 20-28. https://doi.org/10.1016/j.scitotenv.2013.01.038.

Chen, Y., Vymazal, J., Březinová, T., Koželuh, M., Kule, L., Huang, J., Chen, Z., 2016. Occurrence, removal and environmental risk assessment of pharmaceuticals and personal care products in rural wastewater treatment wetlands. Sci. Total Environ. 566-567, 1660-1669. https://doi.org/10.1016/j.scitotenv.2016.06.069.

Chen, J., Meng, T., Li, Y., Gao, K., Qin, Z., 2018. Effects of triclosan on gonadal differentiation and development in the frog Pelophylax nigromaculatus. J. Environ. Sci. (China) 64, 157-165. https://doi.org/10.1016/j.jes.2017.05.040.

Chen, Z.F., Wen, H.B., Dai, X., Yan, S.C., Zhang, H., Chen, Y.Y., Du, Z., Liu, G., Cai, Z., 2018. Contamination and risk profiles of triclosan and triclocarban in sediments from a less urbanized region in China. J. Hazard. Mater. 357, 376-383. https://doi.org/10.1016/j.jhazmat.2018.06.020

Cheng, D., Liu, X., Wang, L., Gong, W., Liu, G., Fu, W., Cheng, M., 2014. Seasonal variation and sediment-water exchange of antibiotics in a shallower large lake in North China. Sci. Total Environ. 476-477, 266-275. https://doi.org/10.1016/j. scitotenv. 2014.01.010
Correia, B., Freitas, R., Figueira, E., Soares, A.M.V.M., Nunes, B., 2016. Oxidative effects of the pharmaceutical drug paracetamol on the edible clam Ruditapes philippinarum under different salinities. Comp. Biochem. Physiol. Part - C Toxicol. Pharmacol. 179, 116-124. https://doi.org/10.1016/j.cbpc.2015.09.006.

Dafouz, R., Cáceres, N., Rodríguez-Gil, J.L., Mastroianni, N., López de Alda, M., Barceló, D., de Miguel, Á.G., Valcárcel, Y., 2018. Does the presence of caffeine in the marine environment represent an environmental risk? A regional and global study. Sci. Total Environ. 615, 632-642. https://doi.org/10.1016/j.scitotenv.2017.09.155.

Dante, G., Vaccaro, F., Facchinetti, F., 2013. Use of progestagens during early pregnancy. FVV ObGyn 5, 66-71.

de Solla, S.R., Gilroy, A.M., Klinck, J.S., King, L.E., McInnis, R., Struger, J., Backus, S.M., Gillis, P.L., 2016. Bioaccumulation of pharmaceuticals and personal care products in the unionid mussel Lasmigona costata in a river receiving wastewater effluent. Chemosphere 146, 486-496. https://doi.org/10.1016/j.chemosphere.2015. 12.022 .

Del Arco, A.I., Parra, G., Rico, A., Van den Brink, P.J., 2015. Effects of intra- and interspecific competition on the sensitivity of aquatic macroinvertebrates to carbendazim. Ecotoxicol. Environ. Saf. 120, 27-34. https://doi.org/10.1016/j.ecoenv. 2015.05.001

Deng, C., Pan, X., Zhang, D., 2015. Influence of ofloxacin on photosystems I and II activities of Microcystis aeruginosa and the potential role of cyclic electron flow. J. Biosci. Bioeng. 119, 159-164. https://doi.org/10.1016/j.jbiosc.2014.07.014.

Deng, W., Li, N., Zheng, H., Lin, H., 2016. Occurrence and risk assessment of antibiotics in river water in Hong Kong. Ecotoxicol. Environ. Saf. 125, 121-127. https:// doi.org/10.1016/j.ecoenv.2015.12.002.

Dhaini, H.R., Nassif, R.M., 2014. Exposure assessment of endocrine disruptors in bottled drinking water of Lebanon. Environ. Monit. Assess. 186, 5655-5662. https:// doi.org/10.1007/s10661-014-3810-x.

Diao, P., Chen, Q., Wang, R., Sun, D., Cai, Z., Wu, H., Duan, S., 2017. Phenolic endocrine-disrupting compounds in the Pearl River Estuary: occurrence, bioaccumulation and risk assessment. Sci. Total Environ. 584-585, 1100-1107. https://doi. org/10.1016/j.scitotenv.2017.01.169.

Ding, H., Wu, Y., Zhang, W., Zhong, J., Lou, Q., Yang, P., Fang, Y., 2017. Occurrence, distribution, and risk assessment of antibiotics in the surface water of Poyang Lake, the largest freshwater lake in China. Chemosphere 184, 137-147. https://doi.org/10.1016/j.chemosphere.2017.05.148.

Ding, T., Lin, K., Yang, B., Yang, M., Li, J., Li, W., Gan, J., 2017. Biodegradation of naproxen by freshwater algae Cymbella sp. and Scenedesmus quadricauda and the comparative toxicity. Bioresour. Technol. 238, 164-173. https://doi.org/10.1016/j. biortech.2017.04.018.

Ding, T., Yang, M., Zhang, J., Yang, B., Lin, K., Li, J., Gan, J., 2017. Toxicity, Degradation and Metabolic Fate of Ibuprofen on Freshwater Diatom Navicula sp. https:/ doi.org/10.1016/j.jhazmat.2017.02.004.

Du, J., Zhao, H., Liu, S., Xie, H., Wang, Y., Chen, J., 2017. Antibiotics in the coastal water of the South Yellow Sea in China: occurrence, distribution and ecological risks. Sci. Total Environ. 595, 521-527. https://doi.org/10.1016/j.scitotenv.2017. 03.281 .

Dvořáková Březinova, T., Vymazal, J., Koželuh, M., Kule, L., 2018. Occurrence and Removal of Ibuprofen and its Metabolites in Full-scale Constructed Wetlands Treating Municipal Wastewater. 120, 1-5. https://doi.org/10.1016/j.ecoleng.2018. 05.020 .

Efeoglu, P., Daglioglu, N., Hilal, A., Yaldiz, F., Korkut Gulmen, M., 2013. Determination of cocaine and its major metabolite benzoylecgonine in rabbit hair by GC/MS. Rom. J. Leg. Med. 21, 111-114. https://doi.org/10.4323/rjlm.2013.111.

Elliott, S.M., Brigham, M.E., Kiesling, R.L., Schoenfuss, H.L., Jorgenson, Z.G., 2018. Environmentally Relevant Chemical Mixtures of Concern in Waters of United States Tributaries to the Great Lakes. vol. 14, 509-518. https://doi.org/10.1002/ ieam. 4041 .

EPA, 2017. Atrazine - Background and Updates [WWW Document].

EPA, 2017. Triclosan [WWW Document]

Erhart, B., Chan, P.J., Patton, W.C., King, A., 1998. Ofloxacin: the next generation of antibiotic in sperm and embryo cultures for assisted reproductive technologies. Fertil. Steril. 69, 246-251. https://doi.org/10.1016/S0015-0282(97)00485-8.

Estrada-Arriaga, E.B., Cortés-Muñoz, J.E., González-Herrera, A., Calderón-Mólgora, C.G., de Lourdes Rivera-Huerta, M., Ramírez-Camperos, E., Montellano-Palacios, L., Gelover-Santiago, S.L., Pérez-Castrejón, S., Cardoso-Vigueros, L., Martín-Domínguez, A., García-Sánchez, L., 2016. Assessment of full-scale biological nutrient removal systems upgraded with physico-chemical processes for the removal of emerging pollutants present in wastewaters from Mexico. Sci. Total Environ. 571, 1172-1182. https://doi.org/10.1016/j.scitotenv.2016.07.118.

Falfushynska, H.I., Gnatyshyna, L.L., Horyn, O., Stoliar, O.B., 2017. Vulnerability of marsh frog Pelophylax ridibundus to the typical wastewater effluents ibuprofen, triclosan and estrone, detected by multi-biomarker approach. Comp. Biochem. Physiol. Part C Toxicol. Pharmacol. 202, 26-38. https://doi.org/10.1016/j.cbpc 2017.07.004.

Fawell, J., Nieuwenhuijsen, M.J., 2003. Contaminants in drinking water. Br. Med. Bull. 68, 199-208. https://doi.org/10.1093/bmb/ldg027. 
Ferrando-Climent, L., Collado, N., Buttiglieri, G., Gros, M., Rodriguez-Roda, I., Rodriguez-Mozaz, S., Barceló, D., 2012. Comprehensive study of ibuprofen and its metabolites in activated sludge batch experiments and aquatic environment. Sci. Total Environ. 438, 404-413. https://doi.org/10.1016/j.scitotenv.2012.08.073.

Fingler, S., Mendaš, G., Dvoršćak, M., Stipičević, S., Vasilić, Drevenkar, V., 2017. Herbicide micropollutants in surface, ground and drinking waters within and near the area of Zagreb, Croatia. Environ. Sci. Pollut. Res. 24, 11017-11030. doi:https: //doi.org/10.1007/s11356-016-7074-6.

Fisch, K., Waniek, J.J., Schulz-bull, D.E., 2017. Occurrence of pharmaceuticals and UV- filters in riverine run-o ff s and waters of the German Baltic Sea. Mar. Pollut. Bull. 124, 388-399. https://doi.org/10.1016/j.marpolbul.2017.07.057.

Gabarrón, S., Gernjak, W., Valero, F., Barceló, A., Petrovic, M., Rodríguez-roda, I., 2016. Evaluation of emerging contaminants in a drinking water treatment plant using electrodialysis reversal technology. J. Hazard. Mater. 309, 192-201. https:// doi.org/10.1016/j.jhazmat.2016.02.015.

García-Cambero, J.P., García-Cortés, H., Valcárcel, Y., Catalá, M., 2015. Environmental concentrations of the cocaine metabolite benzoylecgonine induced sublethal toxicity in the development of plants but not in a zebrafish embryo-larval model. J. Hazard. Mater. 300, 866-872. https://doi.org/10.1016/j.jhazmat.2015.08.019.

Garcia-Morales, R., Rodríguez-Delgado, M., Gomez-Mariscal, K., Orona-Navar, C., Hernandez-Luna, C., Torres, E., ... \& Ornelas-Soto, N. 2015. Biotransformation of endocrine-disrupting compounds in groundwater: bisphenol A, nonylphenol, ethynylestradiol and triclosan by a laccase cocktail from Pycnoporus sanguineus CS43. Water, Air, Soil Pollution. 226(8), 251. doi:https://doi.org/10.1007/s11270015-2514-3.

Goff, A.D., Saranjampour, P., Ryan, L.M., Hladik, M.L., Covi, J.A., Armbrust, K.L., Brander, S.M., 2017. The effects of fipronil and the photodegradation product fipronil desulfinyl on growth and gene expression in juvenile blue crabs, Callinectes sapidus, at different salinities. Aquat. Toxicol. 186, 96-104. https://doi.org 10.1016/j.aquatox.2017.02.027.

González-Alonso, S., Merino, L.M., Esteban, S., López de Alda, M., Barceló, D., Durán, J.J., López-Martínez, J., Aceña, J., Pérez, S., Mastroianni, N., Silva, A., Catalá, M., Valcárcel, Y., 2017. Occurrence of pharmaceutical, recreational and psychotropic drug residues in surface water on the northern Antarctic Peninsula region. Environ. Pollut. 229, 241-254. https://doi.org/10.1016/j.envpol.2017.05.060.

González-Pérez, B.K., Sarma, S.S.S., Castellanos-Páez, M.E., Nandini, S., 2018. Multigenerational effects of triclosan on the demography of Plationus patulus and Brachionus havanaensis (ROTIFERA). Ecotoxicol. Environ. Saf. 147, 275-282. https://doi.org/10.1016/j.ecoenv.2017.08.049.

Gracia-Lor, E., Rousis, N.I., Zuccato, E., Bade, R., Baz-Lomba, J.A., Castrignanò, E., Causanilles, A., Hernández, F., Kasprzyk-Hordern, B., Kinyua, J., McCall, A.K., van Nuijs, A.L.N., Plósz, B.G., Ramin, P., Ryu, Y., Santos, M.M., Thomas, K., de Voogt, P., Yang, Z., Castiglioni, S., 2017. Estimation of caffeine intake from analysis of caffeine metabolites in wastewater. Sci. Total Environ. 609, 1582-1588. https://doi.org/10.1016/j.scitotenv.2017.07.258.

Gripp, H.S., Freitas, J.S., Almeida, E.A., Bisinoti, M.C., Moreira, A.B., 2017. Biochemical effects of fipronil and its metabolites on lipid peroxidation and enzymatic antioxidant defense in tadpoles (Eupemphix nattereri: Leiuperidae). Ecotoxicol. Environ. Saf. 136, 173-179. https://doi.org/10.1016/j.ecoenv.2016.10.027.

Guiloski, I.C., Ribas, J.L.C., Piancini, L.D.S., Dagostim, A.C., Cirio, S.M., Fávaro, L.F., Boschen, S.L., Cestari, M.M., da Cunha, C., Silva de Assis, H.C., 2017. Paracetamol causes endocrine disruption and hepatotoxicity in male fish Rhamdia quelen after subchronic exposure. Environ. Toxicol. Pharmacol. 53, 111-120. https://doi.org/10.1016/j.etap.2017.05.005.

Guiloski, I.C., Stein Piancini, L.D., Dagostim, A.C., de Morais Calado, S.L., Fávaro, L.F., Boschen, S.L., Cestari, M.M., da Cunha, C., Silva de Assis, H.C., 2017. Effects of environmentally relevant concentrations of the anti-inflammatory drug diclofenac in freshwater fish Rhamdia quelen. Ecotoxicol. Environ. Saf. 139, 291-300. https://doi.org/10.1016/j.ecoenv.2017.01.053.

He, S., Dong, D., Zhang, X., Sun, C., Wang, C., Hua, X., Zhang, L., Guo, Z., 2018. Occurrence and Ecological Risk Assessment of 22 Emerging Contaminants in the Jilin Songhua River (Northeast China). 24003-24012.

He, S., Dong, D., Sun, C., Zhang, X., Zhang, L., Hua, X., Guo, Z., 2019. Contaminants of emerging concern in a freeze-thaw river during the spring fl ood. Sci. Total Environ. 670, 576-584. https://doi.org/10.1016/j.scitotenv.2019.03.256.

Hong, H.N., Kim, H.N., Park, K.S., Lee, S.-K., Gu, M.B., 2007. Analysis of the effects diclofenac has on Japanese medaka (Oryzias latipes) using real-time PCR. Chemosphere 67, 2115-2121. https://doi.org/10.1016/j.chemosphere.2006.12.090.

Honjo, A., Arimura, R., Oliveira, L., Pereira, J., Rodriguez de Azevedo, J., 2017. Occurrence of pharmaceutical products, female sex hormones and caffeine in a subtropical region in Brazil. Clean 45, https://doi.org/10.1002/clen.201700334.

Hossain, A., Nakamichi, S., Tani, K., 2018. Occurrence and ecological risk of pharmaceuticals in river surface water of Bangladesh. Environ. Res. 165, 258-266. https:// doi.org/10.1016/j.envres.2018.04.030.

Hou, L., Xu, H., Ying, G., Yang, Y., Shu, H., Zhao, J., Cheng, X., 2017. Physiological responses and gene expression changes in the western mosquitofish (Gambusia affinis) exposed to progesterone at environmentally relevant concentrations. Aquat. Toxicol. 192, 69-77. https://doi.org/10.1016/j.aquatox.2017.09.011.

Huang, F., Zou, S., Deng, D., Lang, H., Liu, F., 2019. Antibiotics in a typical karst river system in China: spatiotemporal variation and environmental risks. Sci. Total Environ. 650, 1348-1355. https://doi.org/10.1016/j.scitotenv.2018.09.131.

Ibe, K., Sim, W., Lee, H., Oh, J., 2018. Occurrence and distribution of carbamazepine, nicotine, estrogenic compounds, and their transformation products in wastewater from various treatment plants and the aquatic environment. Sci. Total Environ. 640-641, 1015-1023. https://doi.org/10.1016/j.scitotenv.2018.05.218.

Inostroza, P.A., Massei, R., Wild, R., Krauss, M., Brack, W., 2017. Chemical activity and distribution of emerging pollutants: insights from a multi-compartment analysis of a freshwater system. Environ. Pollut. 231, 339-347. https://doi.org/10.1016/ j.envpol.2017.08.015.

Isidori, M., Lavorgna, M., Nardelli, A., Parrella, A., Previtera, L., Rubino, M., 2005. Ecotoxicity of naproxen and its phototransformation products. Sci. Total Environ. 348, 93-101. https://doi.org/10.1016/j.scitotenv.2004.12.068.

Jiang, J., Wu, S., Wu, C., An, X., Cai, L., Zhao, X., 2014. Embryonic exposure to carbendazim induces the transcription of genes related to apoptosis, immunotoxicity and endocrine disruption in zebrafish (Danio rerio). Fish Shellfish Immunol 41, 493-500. https://doi.org/10.1016/j.fsi.2014.09.037.

Jiang, J., Wu, S., Wang, Y., An, X., Cai, L., Zhao, X., Wu, C., 2015. Carbendazim has the potential to induce oxidative stress, apoptosis, immunotoxicity and endocrine disruption during zebrafish larvae development. Toxicol. Vitr. 29, 1473-1481. https://doi.org/10.1016/j.tiv.2015.06.003.

Kazemi, S., Mousavi Kani, S.N., Rezazadeh, L., Pouramir, M., Ghasemi-Kasman, M., Moghadamnia, A.A., 2017. Low dose administration of bisphenol A induces liver toxicity in adult rats. Biochem. Biophys. Res. Commun. 494, 107-112. https://doi. org/10.1016/j.bbrc.2017.10.074

Kermia, A.E.B., Fouial-Djebbar, D., Trari, M., 2016. Occurrence, fate and removal efficiencies of pharmaceuticals in wastewater treatment plants (WWTPs) discharging in the coastal environment of Algiers. Comptes Rendus Chim 19, 963-970. https://doi.org/10.1016/j.crci.2016.05.005.

Kim, H., Lee, I., Oh, J., 2017. Human and veterinary pharmaceuticals in the marine environment including fi sh farms in Korea. Sci. Total Environ. 579, 940-949. https: //doi.org/10.1016/j.scitotenv.2016.10.039.

Klosterhaus, S.L., Grace, R., Hamilton, M.C., Yee, D., 2013. Method validation and reconnaissance of pharmaceuticals, personal care products, and alkylphenols in surface waters, sediments, and mussels in an urban estuary. Environ. Int. 54 92-99. https://doi.org/10.1016/j.envint.2013.01.009.

Kot-Wasik, A., Jakimska, A., Śliwka-Kaszyńska, M., 2016. Occurrence and seasonal variations of 25 pharmaceutical residues in wastewater and drinking water treatment plants. Environ. Monit. Assess. 188, https://doi.org/10.1007/s10661-0165637-0.

Krizman-matasic, I., Kostanjevecki, P., Ahel, M., Terzic, S., 2018. Simultaneous analysis of opioid analgesics and their metabolites in municipal wastewaters and river water by liquid chromatography - tandem mass spectrometry. J. Chromatogr. A 1533, 102-111. https://doi.org/10.1016/j.chroma.2017.12.025.

Kyzar, E., Stewart, A.M., Landsman, S., Collins, C., Gebhardt, M., Robinson, K., Kalueff, A.V., 2013. Behavioral effects of bidirectional modulators of brain monoamines reserpine and d-amphetamine in zebrafish. Brain Res. 1527, 108-116. https://doi.org/10.1016/j.brainres.2013.06.033.

Lai, W.W., Lin, Y., Wang, Y., Guo, Y.L., Lin, A.Y., 2018. Occurrence of Emerging Contaminants in Aquaculture Waters: Cross-Contamination between Aquaculture Systems and Surrounding Waters.

Lardy-Fontan, S., Le Diouron, V., Drouin, C., Lalere, B., Vaslin-Reimann, S., Dauchy, X., Rosin, C., 2017. Validation of a method to monitor the occurrence of 20 relevant pharmaceuticals and personal care products in 167 bottled waters. Sci. Total Environ. 587-588, 118-127. https://doi.org/10.1016/j.scitotenv.2017.02.074.

Lashein, F.E.-D.M., Seleem, A.A., Ahmed, A.A., 2016. Effect of caffeine and retinoic acid on skeleton of mice embryos. J. Basic Appl. Zool. 75, 36-45. https://doi.org/ 10.1016/j.jobaz.2016.06.003.

Lee, J., Ji, K., Lim Kho, Y., Kim, P., Choi, K., 2011. Chronic exposure to diclofenac on two freshwater cladocerans and Japanese medaka. Ecotoxicol. Environ. Saf. 74 1216-1225. https://doi.org/10.1016/j.ecoenv.2011.03.014.

Letsinger, S., Kay, P., Rodríguez-mozaz, S., Villagrassa, M., Barceló, D., Rotchell, J.M., 2019. Spatial and Temporal Occurrence of Pharmaceuticals in UK Estuaries. 678, 74-84. https://doi.org/10.1016/j.scitotenv.2019.04.182.

Li, Q., Peng, S., Sheng, Z., Wang, Y., 2010. Ofloxacin induces oxidative damage to joint chondrocytes of juvenile rabbits: excessive production of reactive oxygen species, lipid peroxidation and DNA damage. Eur. J. Pharmacol. 626, 146-153. https://doi.org/10.1016/j.ejphar.2009.09.044

Li, X., Ying, G.G., Zhao, J.L., Chen, Z.F., Lai, H.J., Su, H.C., 2013. 4-Nonylphenol, bisphenol-A and triclosan levels in human urine of children and students in China, and the effects of drinking these bottled materials on the levels. Environ. Int. 52, 81-86. https://doi.org/10.1016/j.envint.2011.03.026.

Li, H., du, H., Fang, L., Dong, Z., Guan, S., Fan, W., Chen, Z., 2016. Residues and dissipation kinetics of carbendazim and diethofencarb in tomato (Lycopersicon escu- 
lentum Mill.) and intake risk assessment. Regul. Toxicol. Pharmacol. 77, 200-205. https://doi.org/10.1016/j.yrtph.2016.03.012.

Lin, T., Yu, S., Chen, W., 2016. Occurrence, removal and risk assessment of pharmaceutical and personal care products (PPCPs) in an advanced drinking water treatment plant (ADWTP) around Taihu Lake in China. Chemosphere 152, 1-9. https:// doi.org/10.1016/j.chemosphere.2016.02.109.

Lin, Y., Gu, H., Jiang, L., Xu, W., Liu, C., Li, Y., Qian, X., Li, D., Li, Z., Hu, J., Zhang, H., Guo, W., Zhao, Y., Cen, X., 2017. Cocaine modifies brain lipidome in mice. Mol. Cell. Neurosci. 85, 29-44. https://doi.org/10.1016/j.men.2017.08.004.

Lin, H., Chen, L., Li, H., Luo, Z., Lu, J., Yang, Z., 2018. Pharmaceutically active compounds in the Xiangjiang River, China: distribution pattern, source apportionment, and risk assessment. Sci. Total Environ. 636, 975-984. https://doi.org/10.1016/j. scitotenv.2018.04.267.

Lin, H., Li, H., Chen, L., Li, L., Yin, L., Lee, H., Yang, Z., 2018. Mass loading and emission of thirty-seven pharmaceuticals in a typical municipal wastewater treatment plant in Hunan Province, Southern China. Ecotoxicol. Environ. Saf. 147, 530-536. https://doi.org/10.1016/j.ecoenv.2017.08.052.

Liu, S., Chen, H., Zhou, G.J., Liu, S.S., Yue, W.Z., Yu, S., Sun, K.F., Cheng, H., Ying, G.G., Xu, X.R., 2015. Occurrence, source analysis and risk assessment of androgens, glucocorticoids and progestagens in the Hailing Bay region, South China Sea. Sci. Total Environ. 536, 99-107. https://doi.org/10.1016/j.scitotenv.2015.07. 028 .

Liu, Yan-hua, Zhang, S., Ji, G., Wu, S., Guo, R., Cheng, J., Yan, Z., Chen, J., 2017. Occurrence, distribution and risk assessment of suspected endocrine-disrupting chemicals in surface water and suspended particulate matter of Yangtze River (Nanjing section). Ecotoxicol. Environ. Saf. 135, 90-97. https://doi.org/10.1016/j. ecoenv.2016.09.035.

Liu, Yanhua, Zhang, S., Song, N., Guo, R., Chen, M., Mai, D., Yan, Z., Han, Z., Chen, J., 2017. Science of the Total Environment Occurrence, distribution and sources of bisphenol analogues in a shallow Chinese freshwater lake (Taihu Lake): implications for ecological and human health risk. Sci. Total Environ. 599-600, 1090-1098. https://doi.org/10.1016/j.scitotenv.2017.05.069.

López-Doval, J.C., Montagner, C.C., de Alburquerque, A.F., Moschini-Carlos, V., Umbuzeiro, G., Pompêo, M., 2017. Nutrients, emerging pollutants and pesticides in a tropical urban reservoir: spatial distributions and risk assessment. Sci. Total Environ. 575, 1307-1324. https://doi.org/10.1016/j.scitotenv.2016.09.210.

López-Pacheco, I.Y., Carrillo-Nieves, D., Salinas-Salazar, C., Silva-Núñez, A., Arévalo-Gallegos, A., Barceló, D., ... Parra-Saldívar, R., 2019. Combination of nejayote and swine wastewater as a medium for Arthrospira maxima and Chlorella vulgaris production and wastewater treatment. Sci. Total Environ. 676, 356-367. https://doi.org/10.1016/j.scitotenv.2019.04.278

Lyndall, J., Barber, T., Mahaney, W., Bock, M., Capdevielle, M., 2017. Evaluation of triclosan in Minnesota lakes and rivers: part I - ecological risk assessment. Ecotoxicol. Environ. Saf. 142, 578-587. https://doi.org/10.1016/j.ecoenv.2017.04.049.

Ma, L., Liu, Y., Zhang, J., Yang, Q., Li, G., Zhang, D., 2018. Impacts of irrigation water sources and geochemical conditions on vertical distribution of pharmaceutical and personal care products (PPCPs) in the vadose zone soils. Sci. Total Environ. 626, 1148-1156. https://doi.org/10.1016/j.scitotenv.2018.01.168.

Ma, X., Wan, Y., Wu, M., Xu, Y., Xu, Q., He, Z., Xia, W., 2018. Occurrence of benzophenones, parabens and triclosan in the Yangtze River of China, and the implications for human exposure. Chemosphere 213, 517-525. https://doi.org/10.1016/j. chemosphere.2018.09.084

Ma, W.L., Zhao, X., Zhang, Z.F., Xu, T.F., Zhu, F.J., Li, Y.F., 2018. Concentrations and fate of parabens and their metabolites in two typical wastewater treatment plants in northeastern China. Sci. Total Environ. 644, 754-761. https://doi.org/10. 1016/j.scitotenv.2018.06.358.

Mac Loughlin, C., Canosa, I.S., Silveyra, G.R., López Greco, L.S., Rodríguez, E.M., 2016. Effects of atrazine on growth and sex differentiation, in juveniles of the freshwater crayfish Cherax quadricarinatus. Ecotoxicol. Environ. Saf. 131, 96-103. https://doi.org/10.1016/j.ecoenv.2016.05.009.

Maguire, R., Kunc, M., Hyrsl, P., Kavanagh, K., 2017. Caffeine administration alters the behaviour and development of Galleria mellonella larvae. Neurotoxicol. Teratol. 64, 37-44. https://doi.org/10.1016/J.NTT.2017.10.002.

Mandaric, L., Diamantini, E., Stella, E., Cano-paoli, K., Valle-sistac, J., Molins-delgado, D., Bellin, A., Chiogna, G., Majone, B., Diaz-cruz, M.S., Sabater, S., Barcelo, D., Petrovic, M., 2017. Contamination sources and distribution patterns of pharmaceuticals and personal care products in Alpine rivers strongly affected by tourism. Sci. Total Environ. 590-591, 484-494. https://doi.org/10.1016/j.scitotenv 2017.02.185.

Marks, J., Bondi, C., Wroblewski, L., Raatikainen, H., Lenox, S., Gebhardt, K., 2015. Human and environmental toxicity of sodium lauryl sulfate (SLS): evidence for safe use in household cleaning products. Environ. Health Insights 27, https://doi org/10.4137/EHI.S31765.

Marsik, P., Rezek, J., Zidkov, M., Kramulov, B., Tauchen, J., Vanek, T., 2017. Non-steroidal Anti-inflammatory Drugs in the Watercourses of Elbe Basin in Czech Republic. 171. https://doi.org/10.1016/j.chemosphere.2016.12.055.
Meador, J.P., Yeh, A., Gallagher, E.P., 2017. Determining potential adverse effects in marine fish exposed to pharmaceuticals and personal care products with the fish plasma model and whole-body tissue concentrations. Environ. Pollut. 230, 1018-1029. https://doi.org/10.1016/j.envpol.2017.07.047.

Merel, S., Benzing, S., Gleiser, C., Napoli-davis, G. Di, Zwiener, C., 2018. Occurrence and overlooked sources of the biocide carbendazim in wastewater and surface water*. Environ. Pollut. 239, 512-521. https://doi.org/10.1016/j.envpol.2018.04.040.

Michel, N., Freese, M., Brinkmann, M., Pohlmann, J.D., Hollert, H., Kammann, U. Haarich, M., Theobald, N., Gerwinski, W., Rotard, W., Hanel, R., 2016. Fipronil and two of its transformation products in water and European eel from the river Elbe. Sci. Total Environ. 568, 171-179. https://doi.org/10.1016/j.scitotenv.2016. 05.210 .

Moeder, M., Carranza-Diaz, O., López-Angulo, G., Vega-Aviña, R., Chávez-Durán, F.A., Jomaa, S., Winkler, U., Schrader, S., Reemtsma, T., Delgado-Vargas, F., 2017. Potential of vegetated ditches to manage organic pollutants derived from agricultural runoff and domestic sewage: a case study in Sinaloa (Mexico). Sci Total Environ. 598, 1106-1115. https://doi.org/10.1016/j.scitotenv.2017.04.149.

Morgan, M.K., Nash, M., Barr, D.B., Starr, J.M., Scott Clifton, M., Sobus, J.R., 2018 Distribution, variability, and predictors of urinary bisphenol A levels in 50 North Carolina adults over a six-week monitoring period. Environ. Int. 112, 85-99. https: //doi.org/10.1016/j.envint.2017.12.014.

Morishima, H.O., Okutomi, T., Ishizaki, A., Zhang, Y., Cooper, T.B., 2001. The disposition of benzoylecgonine in maternal and fetal rats. Neurotoxicol. Teratol. 23, 247-253. https://doi.org/10.1016/S0892-0362(01)00136-2.

Moro, I., Matozzo, V., Piovan, A., Moschin, E., Vecchia, F.D., 2014. Morpho-physiological effects of ibuprofen on scenedesmus rubescens. Environ. Toxicol. Pharmacol. 38, 379-387. https://doi.org/10.1016/j.etap.2014.06.005.

Mossa, A.T.H., Heikal, T.M., Omara, E.A.A., 2012. Physiological and histopathological changes in the liver of male rats exposed to paracetamol and diazinon. Asian Pac. J. Trop. Biomed. 2, S1683-S1690. https://doi.org/10.1016/S22211691(12)60478-X.

Mutiyar, P.K., Kumar, S., Kumar, A., 2018. Fate of pharmaceutical active compounds (PhACs) from River Yamuna, India: An ecotoxicological risk assessment approach. Ecotoxicol. Environ. Saf. 150, 297-304. https://doi.org/10.1016/j.ecoenv. 2017.12.041.

Neal, A.E., Moore, P.A., 2017. Mimicking natural systems: changes in behavior as a result of dynamic exposure to naproxen. Ecotoxicol. Environ. Saf. 135, 347-357. https://doi.org/10.1016/j.ecoenv.2016.10.015.

Nguyen, H.T., Thai, P.K., Kaserzon, S.L., Brien, J.W.O., Eaglesham, G., Mueller, J.F., 2018. Assessment of drugs and personal care products biomarkers in the in $\mathrm{fl}$ uent and ef fl uent of two wastewater treatment plants in Ho Chi Minh. Sci. Total Environ. 631-632, 469-475. https://doi.org/10.1016/j.scitotenv.2018.02.309.

NIH, 2017. Bisphenol A (BPA): Your Environment, Your Health | National Library of Medicine [WWW Document].

Niu, S., Zhang, C., 2018. Endocrine disrupting compounds from the source water of the Huai River (Huainan City), China. Arch. Environ. Contam. Toxicol. 74, 471-483. https://doi.org/10.1007/s00244-017-0445-2.

Nuel, M., Laurent, J., Bois, P., Heintz, D., Wanko, A., 2018. Seasonal and ageing effect on the behaviour of 86 drugs in a full-scale surface treatment wetland: removal ef fi ciencies and distribution in plants and sediments. Sci. Total Environ. 615, 1099-1109. https://doi.org/10.1016/j.scitotenv.2017.10.061.

Ocharoen, Y., Boonphakdee, C., Boonphakdee, T., Shinn, A.P., 2018. High levels of the endocrine disruptors bisphenol-A and $17 \beta$-estradiol detected in populations of green mussel, Perna viridis, cultured in the Gulf of Thailand. Aquaculture 497, 348-356. https://doi.org/10.1016/j.aquaculture.2018.07.057

Omar, T.F.T., Aris, A.Z., Yusoff, F.M., Mustafa, S., 2019. Occurrence and level of emerging organic contaminant in fish and mollusk from Klang River estuary, Malaysia and assessment on human health risk. Environ. Pollut. 248, 763-773. https://doi.org/10.1016/j.envpol.2019.02.060.

Paíga, P., Santos, L.H.M.L.M., Ramos, S., Jorge, S., Silva, J.G., Delerue-Matos, C., 2016. Presence of pharmaceuticals in the Lis river (Portugal): sources, fate and seasonal variation. Sci. Total Environ. 573, 164-177. https://doi.org/10.1016/j. scitotenv.2016.08.089.

Palmiotto, M., Castiglioni, S., Zuccato, E., Manenti, A., Riva, F., Davoli, E., 2018. Personal care products in surface, ground and wastewater of a complex aquifer system, a potential planning tool for contemporary urban settings. J. Environ. Manag. 214, 76-85. https://doi.org/10.1016/j.jenvman.2017.10.069.

Parolini, M., Pedriali, A., Riva, C., Binelli, A., 2013. Sub-lethal effects caused by the cocaine metabolite benzoylecgonine to the freshwater mussel Dreissena polymorpha. Sci. Total Environ. 444, 43-50. https://doi.org/10.1016/j.scitotenv.2012.11. 076.

Parolini, M., Bini, L., Magni, S., Rizzo, A., Ghilardi, A., Landi, C., Armini, A., Giacco, L. Del, Binelli, A., 2017. Exposure to Cocaine and its Main Metabolites Altered the Protein Profile of Zebrafish Embryos. 232, 603-614. https://doi.org/10. 1016/j.envpol.2017.09.097.

Parolini, M., De Felice, B., Ferrario, C., Salgueiro-González, N., Castiglioni, S., Finizio, A., Tremolada, P., 2017. Benzoylecgonine exposure induced oxidative 
stress and altered swimming behavior and reproduction in Daphnia magna. Environ. Pollut. 232, 236-244. https://doi.org/10.1016/j.envpol.2017.09.038.

Paulo, A.M.S., Aydin, R., Dimitrov, M.R., Vreeling, H., Cavaleiro, A.J., García-Encina, P.A., Stams, A.J.M., Plugge, C.M., 2017. Sodium lauryl ether sulfate (SLES) degradation by nitrate-reducing bacteria. Appl. Microbiol. Biotechnol 101, 5163-5173. https://doi.org/10.1007/s00253-017-8212-x.

Pavlić, , Vidaković-Cifrek, , Puntarić, D., 2005. Toxicity of surfactants to green microalgae Pseudokirchneriella subcapitata and Scenedesmus subspicatus and to marine diatoms Phaeodactylum tricornutum and Skeletonema costatum. Chemosphere 61, 1061-1068. https://doi.org/10.1016/j.chemosphere.2005.03.051.

Peltzer, P.M., Lajmanovich, R.C., Martinuzzi, C., Attademo, A.M., Curi, L.M., Sandoval, M.T., 2019. Biotoxicity of diclofenac on two larval amphibians: assessmen of development, growth, cardiac function and rhythm, behavior and antioxidant system. Sci. Total Environ. 683, 624-637. https://doi.org/10.1016/j.scitotenv.2019. 05.275 .

Peng, Y., Fang, W., Krauss, M., Brack, W., Wang, Z., Li, F., Zhang, X., 2018. Screening hundreds of emerging organic pollutants (EOPs) in surface water from the Yangtze River Delta (YRD): occurrence, distribution, ecological risk. Environ. Pollut. 241, 484-493. https://doi.org/10.1016/j.envpol.2018.05.061.

Pereira, A.M.P.T., Silva, L.J.G., Laranjeiro, C.S.M., Meisel, L.M., Lino, C.M., Pena, A., 2017. Human pharmaceuticals in Portuguese rivers: the impact of water scarcity in the environmental risk. Sci. Total Environ. 609, 1182-1191. https://doi. org/10.1016/j.scitotenv.2017.07.200.

Plahuta, M., Tišler, T., Pintar, A., Toman, M.J., 2015. Adverse effects of bisphenol A on water louse (Asellus aquaticus). Ecotoxicol. Environ. Saf. 117, 81-88. https:// doi.org/10.1016/j.ecoenv.2015.03.031.

Poi, C. Di, Costil, K., Bouchart, V., 2018. Toxicity Assessment of Five Emerging Pollutants, Alone and in Binary or Ternary Mixtures, towards Three Aquatic Organisms. 6122-6134. https://doi.org/10.1007/s11356-017-9306-9.

Primrose, N., Naicker, D., Ncube, S., Chimuka, L., 2019. Determination of naproxen, diclofenac and ibuprofen in Umgeni estuary and seawater: a case of northern Durban in KwaZulu - Natal Province of South Africa. Reg. Stud. Mar. Sci. 29, 100675https://doi.org/10.1016/j.rsma.2019.100675.

Radley, P.A.U.L.M.B., Attaglin, W.I.A.B., Lark, J.I.M.C., Enning, F.R.P.H., Ladik, M.I.L.H., Wanowicz, L.U.K.E.R.I., Ourney, C.E.A.J., Iley, J.E.W.R., Omanokf, K.R.M.R., 2017. Widespread occurrence and potential for biodegradation of bioactive contaminants in congaree national park, USA. Environ. Toxicol. Chem. 36, 3045-3056. https://doi.org/10.1002/etc.3873.

Rah, Y.C., Yoo, M.H., Choi, J., Park, S., Park, H.C., Oh, K.H., Lee, S.H., Kwon, S.Y., 2017. In vivo assessment of hair cell damage and developmental toxicity caused by gestational caffeine exposure using zebrafish (Danio rerio) models. Neurotoxicol. Teratol. 64, 1-7. https://doi.org/10.1016/j.ntt.2017.08.003.

Rhind, S.M., 2009. Anthropogenic pollutants: a threat to ecosystem sustainability ? Philos. Trans. R. Soc. 364, 3391-3401. https://doi.org/10.1098/rstb.2009.0122.

Ribeiro de Sousa, D., Mozeto, A., Lajarim, R., Fadini, P., 2018. Spatio-Temporal Evaluation of Emerging Contaminants and their Partitioning Along a Brazilian Watershed. 4607-4620.

Riva, C., Cristoni, S., Binelli, A., 2012. Effects of triclosan in the freshwater mussel Dreissena polymorpha: a proteomic investigation. Aquat. Toxicol. 118-119, 62-71. https://doi.org/10.1016/j.aquatox.2012.03.013.

Riva, F., Castiglioni, S., Fattore, E., Manenti, A., Davoli, E., Zuccato, E., 2018. Monitoring emerging contaminants in the drinking water of Milan and assessment of the human risk. Int. J. Hyg. Environ. Health 221, 451-457. https://doi.org/10.1016/j. ijheh.2018.01.008.

Riva, F., Zuccato, E., Davoli, E., Fattore, E., Castiglioni, S., 2019. Risk assessment of a mixture of emerging contaminants in surface water in a highly urbanized area in Italy. J. Hazard. Mater. 361, 103-110. https://doi.org/10.1016/j.jhazmat.2018.07. 099.

Rivera-Jaimes, J.A., Postigo, C., Melgoza-Alemán, R.M., Aceña, J., Barceló, D., López de Alda, M., 2018. Study of pharmaceuticals in surface and wastewater from Cuernavaca, Morelos, Mexico: occurrence and environmental risk assessment. Sci. Total Environ. 613-614, 1263-1274. https://doi.org/10.1016/j.scitotenv. 2017.09.134

Robledo, P., 2008. Las anfetaminas. Trastor. Adict. 10, 166-174. https://doi.org/10. 1016/S1575-0973(08)76363-3.

Rodríguez-Delgado, M., Orona-Navar, C., García-Morales, R., Hernandez-Luna, C., Parra, R., Mahlknecht, J., Ornelas-Soto, N., 2016. Biotransformation kinetics of pharmaceutical and industrial micropollutants in groundwaters by a laccase cocktail from Pycnoporus sanguineus CS43 fungi. Int. Biodeter. Biodegrad. 108, 34 -41. https://doi.org/10.1016/j.ibiod.2015.12.003.

Sanzi, F., Souza, S., Lopes, L., Emanoel, J., Hermes, F., Alves, L., Gonçalves, L., Rodrigues, C., Barbosa, B., Moledo, D., Abessa, D.S., Cesar, A., Ramos, A., Dias, C. Pereira, S., 2018. Ecotoxicological effects of losartan on the brown mussel Perna perna and its occurrence in seawater from Santos Bay (Brazil). Sci. Total Environ. 637-638, 1363-1371. https://doi.org/10.1016/j.scitotenv.2018.05.069.

Sari, S., Ozdemir, G., Yangin-Gomec, C., Zengin, G.E., Topuz, E., Aydin, E., Pehlivanoglu-Mantas, E., Okutman Tas, D., 2014. Seasonal variation of diclofenac con- centration and its relation with wastewater characteristics at two municipal wastewater treatment plants in Turkey. J. Hazard. Mater. 272, 155-164. https://doi.org/ 10.1016/j.jhazmat.2014.03.015.

Scheurell, M., Franke, S., Shah, R.M., Hühnerfuss, H., 2009. Occurrence of diclofenac and its metabolites in surface water and effluent samples from Karachi, Pakistan. Chemosphere 77, 870-876. https://doi.org/10.1016/j.chemosphere.2009.07.066.

Sehonova, P., Plhalova, L., Blahova, J., Doubkova, V., Prokes, M., Tichy, F., Fiorino, E., Faggio, C., Svobodova, Z., 2017. Toxicity of naproxen sodium and its mixture with tramadol hydrochloride on fish early life stages. Chemosphere 188, 414-423. https://doi.org/10.1016/j.chemosphere.2017.08.151.

Sharma, B.M., Bečanová, J., Scheringer, M., Sharma, A., Bharat, G.K., Whitehead, P.G., Klánová, J., Nizzetto, L., 2019. Health and ecological risk assessment of emerging contaminants (pharmaceuticals, personal care products, and artificial sweeteners) in surface and groundwater (drinking water) in the Ganges River Basin, India. Sci. Total Environ. 646, 1459-1467. https://doi.org/10.1016/j. scitotenv.2018.07.235.

Shi, M., Sekulovski, N., MacLean, J.A., Hayashi, K., 2017. Effects of bisphenol A analogues on reproductive functions in mice. Reprod. Toxicol. 73, 280-291. https: //doi.org/10.1016/j.reprotox.2017.06.134.

Sibila, M.A., Garrido, M.C., Perales, J.A., Quiroga, J.M., 2008. Ecotoxicity and biodegradability of an alkyl ethoxysulphate surfactant in coastal waters. Sci. Total Environ. 394, 265-274. https://doi.org/10.1016/j.scitotenv.2008.01.043.

Silva, B.F. da, Jelic, A., López-Serna, R., Mozeto, A.A., Petrovic, M., Barceló, D., 2011. Occurrence and distribution of pharmaceuticals in surface water, suspended solids and sediments of the Ebro river basin, Spain. Chemosphere 85, 1331-1339. https://doi.org/10.1016/j.chemosphere.2011.07.051.

Silva, A.R.R., Cardoso, D.N., Cruz, A., Lourenço, J., Mendo, S., Soares, A.M.V.M., Loureiro, S., 2015. Ecotoxicity and genotoxicity of a binary combination of triclosan and carbendazim to Daphnia magna. Ecotoxicol. Environ. Saf. 115, 279-290. https://doi.org/10.1016/j.ecoenv.2015.02.022.

Sodré, F.F., Santana, J.S., Sampaio, T.R., Brandão, C.C.S., 2018. Seasonal and spatial distribution of caffeine, atrazine, atenolol and deet in surface and drinking waters from the brazilian federal district. J. Braz. Chem. Soc. 29, 1854-1865. https://doi. org/10.21577/0103-5053.20180061.

Sposito, J.C.V., Montagner, C.C., Casado, M., Navarro-martín, L., Julio, C., 2018. Emerging Contaminants in Brazilian Rivers: Occurrence and Effects on Gene Expression in Zebra Fish (Danio rerio) Embryos. 209, 696-704. https://doi.org/10. 1016/j.chemosphere.2018.06.046.

Stancová, V., Ziková, A., Svobodová, Z., Kloas, W., 2015. Effects of the non-steroidal anti-inflammatory drug(NSAID) naproxen on gene expression of antioxidant enzymes in zebrafish (Danio rerio). Environ. Toxicol. Pharmacol. 40, 343-348. https: //doi.org/10.1016/j.etap.2015.07.009.

Staniszewska, M., Koniecko, I., Falkowska, L., Krzymyk, E., 2015. Occurrence and distribution of bisphenol A and alkylphenols in the water of the gulf of Gdansk (Southern Baltic). Mar. Pollut. Bull. 91, 372-379. https://doi.org/10.1016/j. marpolbul.2014.11.027.

Stark, J.D., Vargas, R.I., 2005. Toxicity and hazard assessment of fipronil to Daphnia pulex. Ecotoxicol. Environ. Saf. 62, 11-16. https://doi.org/10.1016/j.ecoenv.2005. 02.011 .

Stayner, L.T., Almberg, K., Jones, R., Graber, J., Pedersen, M., Turyk, M., 2017. Atrazine and nitrate in drinking water and the risk of preterm delivery and low birth weight in four Midwestern states. Environ. Res. 152, 294-303. https://doi. org/10.1016/j.envres.2016.10.022.

Sui, Q., Zhao, W., Cao, X., Lu, S., Qiu, Z., Gu, X., 2017. Pharmaceuticals and personal care products in the leachates from a typical landfill reservoir of municipal solid waste in Shanghai, China: occurrence and removal by a full-scale membrane bioreactor. J. Hazard. Mater. 323, 99-108. https://doi.org/10.1016/j.jhazmat.2016.03. 047.

Sun, J., Luo, Q., Wang, D., Wang, Z., 2015. Occurrences of pharmaceuticals in drinking water sources of major river watersheds, China. Ecotoxicol. Environ. Saf. 117, 132-140. https://doi.org/10.1016/j.ecoenv.2015.03.032.

Świacka, K., Szaniawska, A., Caban, M., 2019. Evaluation of bioconcentration and metabolism of diclofenac in mussels Mytilus trossulus - laboratory study. Mar. Pollut. Bull. 141, 249-255. https://doi.org/10.1016/j.marpolbul.2019.02.050.

Tan, R., Liu, R., Li, B., Liu, X., Li, Z., 2018. Typical endocrine disrupting compounds in Rivers of Northeast China: occurrence, partitioning, and risk assessment. Arch. Environ. Contam. Toxicol. 75, 213-223. https://doi.org/10.1007/s00244-017-0482$\mathrm{x}$

Teerlink, J., Hernandez, J., Budd, R., 2017. Fipronil washoff to municipal wastewater from dogs treated with spot-on products. Sci. Total Environ. 599-600, 960-966. https://doi.org/10.1016/j.scitotenv.2017.04.219.

Thai, P.K., Ky, L.X., Binh, V.N., Nhung, P.H., Nhan, P.T., Hieu, N.Q., Dang, N.T.T. Tam, N.K.B., Anh, N.T.K., 2018. Occurrence of antibiotic residues and antibiotic-resistant bacteria in effluents of pharmaceutical manufacturers and other sources around Hanoi, Vietnam. Sci. Total Environ. 645, 393-400. https://doi.org/ 10.1016/j.scitotenv.2018.07.126. 
Toan, P. Van, Sebesvari, Z., Bläsing, M., Rosendahl, I., Renaud, F.G., 2013. Pesticide management and their residues in sediments and surface and drinking water in the Mekong Delta, Vietnam. Sci. Total Environ. 452-453, 28-39. https://doi.org/10. 1016/j.scitotenv.2013.02.026.

Valenzuela, A., 2010. El café y sus efectos en la salud cardiovascular y en la salud materna. Rev. Chil. Nutr. 37, 514-523. https://doi.org/10.4067/S071775182010000400013

Van Den Brink, P.J., Hattink, J., Bransen, F., Van Donk, E., Brock, T.C.M., 2000. Impact of the fungicide carbendazim in freshwater microcosms. II. Zooplankton, primary producers and final conclusions. Aquat. Toxicol. 48, 251-264. https://doi. org/10.1016/S0166-445X(99)00037-5.

van Nuijs, A.L.N., Pecceu, B., Theunis, L., Dubois, N., Charlier, C., Jorens, P.G., Bervoets, L., Blust, R., Neels, H., Covaci, A., 2009. Cocaine and metabolites in waste and surface water across Belgium. Environ. Pollut. 157, 123-129. https:// doi.org/10.1016/j.envpol.2008.07.020.

van Nuijs, A.L.N., Pecceu, B., Theunis, L., Dubois, N., Charlier, C., Jorens, P.G., Bervoets, L., Blust, R., Neels, H., Covaci, A., 2009. Spatial and temporal variations in the occurrence of cocaine and benzoylecgonine in waste- and surface water from Belgium and removal during wastewater treatment. Water Res. 43 , 1341-1349. https://doi.org/10.1016/j.watres.2008.12.020.

Van Wijngaarden, R.P.A., Crum, S.J.H., Decraene, K., Hattink, J., Van Kammen, A., 1998. Toxicicity of Derosal (active ingredient carbendazim) to aquatic invertebrates. Chemosphere 37, 673-683. https://doi.org/10.1016/S0045-6535(98)000836.

Vimalkumar, K., Arun, E., Krishna-kumar, S., Poopal, R.K., Nikhil, N.P., Subramanian, A., Babu-rajendran, R., 2018. Occurrence of triclocarban and benzotriazole ultraviolet stabilizers in water, sediment, and fi sh from Indian rivers. Sci. Total Environ. 625, 1351-1360. https://doi.org/10.1016/j.scitotenv.2018.01.042.

Vulliet, E., Cren-Olive, C., 2011. Screening of pharmaceuticals and hormones at the regional scale, in surface and groundwaters intended to human consumption. Environ. Pollut. 159, 2929-2934. https://doi.org/10.1016/j.envpol.2011.04.033.

Wagner, S.D., Kurobe, T., Hammock, B.G., Lam, C.H., Wu, G., Vasylieva, N., Gee, S.J., Hammock, B.D., Teh, S.J., 2017. Developmental effects of fipronil on Japanese Medaka (Oryzias latipes) embryos. Chemosphere 166, 511-520. https://doi. org/10.1016/j.chemosphere.2016.09.069

Wang, L., Ying, G.G., Zhao, J.L., Yang, X.B., Chen, F., Tao, R., Liu, S., Zhou, L.J., 2010. Occurrence and risk assessment of acidic pharmaceuticals in the Yellow River, Hai River and Liao River of north China. Sci. Total Environ. 408, 3139-3147. https://doi.org/10.1016/j.scitotenv.2010.04.047.

Wang, Q., Chen, M., Shan, G., Chen, P., Cui, S., Yi, S., Zhu, L., 2017. Bioaccumulation and biomagnification of emerging bisphenol analogues in aquatic organisms from Taihu Lake, China. Sci. Total Environ. 598, 814-820. https://doi.org/10. 1016/j.scitotenv.2017.04.167.

Wang, Z., Li, X., Klaunig, J.E., 2017. Investigation of the mechanism of triclosan in duced mouse liver tumors. Regul. Toxicol. Pharmacol. 86, 137-147. https://doi. org/10.1016/j.yrtph.2017.03.001

Wang, Yuwen, Li, Y., Hu, A., Rashid, A., Ashfaq, M., Wang, Yinhan, 2018. Monitoring, mass balance and fate of pharmaceuticals and personal care products in seven wastewater treatment plants in Xiamen City, China. J. Hazard. Mater. 354, 81-90. https://doi.org/10.1016/j.jhazmat.2018.04.064.

Wei-po Lai, W., Lin, Y., Tung, H., Lo, S., Yu-chen Lin, A., 2016. Occurrence of pharmaceuticals and per fl uorinated compounds and evaluation of the availability of reclaimed water in Kinmen. Emerg. Contam. 2, 135-144. https://doi.org/10.1016/j. emcon.2016.05.001

Wilkinson, J.L., Hooda, P.S., Swinden, J., Barker, J., Barton, S., 2017. Spatial distribution of organic contaminants in three rivers of Southern England bound to suspended particulate material and dissolved in water. Sci. Total Environ. 593-594, 487-497. https://doi.org/10.1016/j.scitotenv.2017.03.167.

Wirbisky, S.E., Freeman, J.L., 2017. Atrazine exposure elicits copy number alteration in the zebrafish genome. Comp. Biochem. Physiol. Part - C Toxicol. Pharmacol. 194, 1-8. https://doi.org/10.1016/j.cbpc.2017.01.003.

Wirbisky, S.E., Weber, G.J., Schlotman, K.E., Sepúlveda, M.S., Freeman, J.L., 2016. Embryonic atrazine exposure alters zebrafish and human miRNAs associated with angiogenesis, cancer, and neurodevelopment. Food Chem. Toxicol. 98, 25-33. https://doi.org/10.1016/j.fct.2016.03.027.
Wirbisky-Hershberger, S.E., Sanchez, O.F., Horzmann, K.A., Thanki, D., Yuan, C., Freeman, J.L., 2017. Atrazine exposure decreases the activity of DNMTs, global DNA methylation levels, and dnmt expression. Food Chem. Toxicol. 109, 727-734. https://doi.org/10.1016/j.fct.2017.08.041

Wu, J., Lu, J., Lu, H., Lin, Y., Chris Wilson, P., 2015. Occurrence and ecological risks from fipronil in aquatic environments located within residential landscapes. Sci. Total Environ. 518-519, 139-147. https://doi.org/10.1016/j.scitotenv.2014.12.103.

Wu, Q., Lam, J.C.W., Kwok, K.Y., Tsui, M.M.P., Lam, P.K.S., 2016. Occurrence and fate of endogenous steroid hormones, alkylphenol ethoxylates, bisphenol A and phthalates in municipal sewage treatment systems. J. Environ. Sci. (China) 1-10. https://doi.org/10.1016/j.jes.2017.02.021.

Wu, M.H., Que, C.J., Xu, G., Sun, Y.F., Ma, J., Xu, H., Sun, R., Tang, L., 2016. Occurrence, fate and interrelation of selected antibiotics in sewage treatment plants and their receiving surface water. Ecotoxicol. Environ. Saf. 132, 132-139. https:// doi.org/10.1016/j.ecoenv.2016.06.006.

Wu, M., Xiang, J., Chen, F., Fu, C., Xu, G., 2017. Occurrence and Risk Assessment of Antidepressants in Huangpu River of Shanghai, China. 20291-20299. https://doi. org/10.1007/s11356-017-9293-x.

Xia, L., Zheng, L., Zhou, J.L., 2017. Effects of ibuprofen, diclofenac and paracetamol on hatch and motor behavior in developing zebrafish (Danio rerio). Chemosphere 182, 416-425. https://doi.org/10.1016/j.chemosphere.2017.05.054

Xiang, J., Wu, M., Lei, J., Fu, C., Gu, J., Xu, G., 2018. The fate and risk assessment of psychiatric pharmaceuticals from psychiatric hospital e ffl uent. Ecotoxicol. Environ. Saf. 150, 289-296. https://doi.org/10.1016/j.ecoenv.2017.12.049.

Xu, J., Zhang, Y., Zhou, C., Guo, C., Wang, D., Du, P., Luo, Y., Wan, J., Meng, W., 2014. Distribution, sources and composition of antibiotics in sediment, overlying water and pore water from Taihu Lake, China. Sci. Total Environ. 497-498, 267-273. https://doi.org/10.1016/j.scitotenv.2014.07.114

Yadav, M.K., Short, M.D., Gerber, C., Akker, B. Van Den, Aryal, R., 2018. Occurrence, Removal and Environmental Risk of Markers of Five Drugs of Abuse in Urban Wastewater Systems in South Australia.

Yamamoto, F.Y., Diamante, G.D., Santana, M.S., Santos, D.R., Bombardeli, R., Martins, C.C., Ribeiro, C.A.O., Schlenk, D., 2018. Alterations of cytochrome P450 and the occurrence of persistent organic pollutants in tilapia caged in the reservoirs of the Iguaçu. Environ. Pollut. 240, 670-682. https://doi.org/10.1016/j.envpol.2018. 04.019 .

Yamazaki, E., Yamashita, N., Taniyasu, S., Lam, J., Lam, P.K.S., Moon, H.B., Jeong, Y., Kannan, P., Achyuthan, H., Munuswamy, N., Kannan, K., 2015. Bisphenol A and other bisphenol analogues including BPS and BPF in surface water samples from Japan, China, Korea and India. Ecotoxicol. Environ. Saf. 122, 565-572. https://doi.org/10.1016/j.ecoenv.2015.09.029.

Yang, Y., Zhao, J., Liu, Y., Liu, W., Zhang, Q., Yao, L., 2018. Pharmaceuticals and personal care products (PPCPs) and arti fi cial sweeteners (ASs) in surface and ground waters and their application as indication of wastewater contamination. Sci. Total Environ. 616-617, 816-823. https://doi.org/10.1016/j.scitotenv.2017.10.241.

Yao, B., Yan, S., Lian, L., Yang, X., Wan, C., Dong, H., Song, W., 2018. Occurrence and indicators of pharmaceuticals in Chinese streams: a nationwide study *. Environ. Pollut. 236, 889-898. https://doi.org/10.1016/j.envpol.2017.10.032.

Zha, D., Li, Y., Wang, L., Yang, C., Lu, G., 2017. Occurrence and Attenuation of Pharmaceuticals and their Transformation Products in Rivers Impacted by Sewage Treatment Plants. 40905-40913. https://doi.org/10.1039/c7ra06852b.

Zhang, Y., Wang, B., Cagnetta, G., Duan, L., Yang, J., Deng, S., Huang, J., Wang, Y., $\mathrm{Yu}, \mathrm{G} ., 2018$. Typical pharmaceuticals in major WWTPs in Beijing, China: occurrence, load pattern and calculation reliability. Water Res. 140, 291-300. https:// doi.org/10.1016/j.watres.2018.04.056

Zhang, H., Zhang, Y., Li, J., Yang, M., 2019. Occurrence and exposure assessment of bisphenol analogues in source water and drinking water in China. Sci. Total Environ. 655, 607-613. https://doi.org/10.1016/j.scitotenv.2018.11.053.

Zrinyi, Z., Maasz, G., Zhang, L., Vertes, A., Lovas, S., Kiss, T., Elekes, K., Pirger, Z., 2017. Effect of progesterone and its synthetic analogs on reproduction and embryonic development of a freshwater invertebrate model. Aquat. Toxicol. 190 94-103. https://doi.org/10.1016/j.aquatox.2017.06.029. 